\title{
The quantum chiral Minkowski and conformal superspaces
}

\author{
Dalia Cervantes ${ }^{1}$, Rita Fioresi ${ }^{2}$ and María A. Lledó ${ }^{3}$
}

\author{
${ }^{1}$ Instituto de Ciencias Nucleares, \\ Universidad Nacional Autónoma de México, \\ Circuito Exterior, México D.F. 04510, México \\ daliac@nucleares.unam.mx \\ ${ }^{2}$ Dipartimento di Matematica, Università di Bologna, \\ Piazza di Porta S. Donato, 5. 40126 Bologna, Italy \\ fioresi@dm. UniBo.it \\ ${ }^{3}$ Departament de Física Teòrica, \\ Universitat de València and IFIC (CSIC-UVEG) Fundació General, \\ Universitat de València, \\ C/Dr. Moliner, 50, E-46100 Burjassot (València), Spain \\ maria.1ledo@ific.uv.es
}

\begin{abstract}
We give a quantum deformation of the chiral super Minkowski space in four dimensions as the big cell inside a quantum super Grassmannian. The quantization is performed in such way that the actions of the Poincaré and conformal quantum supergroups on the quantum Minkowski and quantum conformal superspaces are preserved.
\end{abstract}

e-print archive: http://lanl.arXiv.org/abs/0811.2137 


\section{Introduction}

The Minkowski space in four dimensions is just $\mathbb{R}^{4}$ with the pseudoeuclidean metric $\operatorname{diag}(1,-1,-1,-1)$. The Poincaré group $\mathrm{P}(1,3)=\mathrm{SO}(1,3) \propto \mathbb{R}^{4}$ is the group that preserves such metric, while the conformal group is the group that preserves the metric up to a global factor. It is in fact the group $\mathrm{SO}(2,4)$ and it acts nonlinearly on a compactification of the Minkowski space, obtained by adjoining to it not just a point at infinity, but the closure of a cone [1]. This compactification turns out to be the Grassmannian manifold $G(2,4)$, that is, the space of 2-planes inside a four-dimensional (4D) vector space and the Poincaré group together with the dilations is precisely the subgroup of $\mathrm{SO}(2,4)$ consisting of the elements that leave the Minkowski space invariant. The rest of the conformal transformations may send a point in the Minkowski space to a point at infinity. The Minkowski space sits inside the Grassmannian $G(2,4)$ as its big cell, which is a dense open set inside it. We will refer to the Grassmannian $G(2,4)$ as the conformal space.

The relation between the Poincaré and conformal group on one side and the Minkowski and conformal space on the other side, are well known, see for example [2]. A brief but complete review can also be found in [3], which we have followed very closely in spirit and notation. The analysis in there starts by considering the spin group of $\mathrm{SO}(2,4)$, which is $\mathrm{SU}(2,2)$, that contains the spin group of $\mathrm{SO}(1,3)$, which is $\mathrm{SL}(2, \mathbb{C})_{\mathbb{R}}$. It is more natural to work in the complexified spaces $(\mathrm{SL}(4, \mathbb{C})$ and $\mathrm{SL}(2, \mathbb{C}) \times \mathrm{SL}(2, \mathbb{C})$, respectively) and to look at the end for the particular real form associated to Minkowskian signature.

This approach is very useful when extending the results to the Minkowski and conformal superspaces (see [1]), since the action on spinors is explicit in the formalism. The fascinating subject of supergeometry emerges here as a very natural framework. Supergeometry extends standard algebraic and differential geometry in a less dramatic way than noncommutative geometry in general [4]. The category of algebras considered in supergeometry are noncommutative, but their noncommutativity affects only to some generators that anticommute. These are the odd generators.

The functor of points is another tool, that one borrows from standard algebraic geometry and extends to the super setting. For a standard algebraic variety, the geometric points are the morphisms from the coordinate ring of the supervariety to the ground field. If one considers the morphisms from the coordinate ring to another commutative ring, say $R$, one has the $R$-points of the algebraic variety. For a supervariety, one takes $R$ to be a commutative superalgebra, and the $R$-points still make sense. The geometric 
points turn out to give the points of a standard variety called the reduced variety, and the odd variables disappear. Only by considering morphisms to superalgebras instead than to commutative algebras or just the ground field one can recover the role of the odd variables, and with it all the information to reconstruct the supervariety.

We then see that via the functor of points the true nature of the odd variables appears in a beautiful, perfectly consistent framework $[3,5,6]$, which is very close to the way in which physicists have been thinking and talking about superspaces and supergroups. The terminology and perhaps the level of rigor becomes more sophisticated, but a closer look reveals many old concepts that have been used implicitly by physicists are at the core of the mathematical formulation.

The next step is to produce a noncommutative version of the Minkowski and conformal superspaces. In order to do this, we need to substitute the commutative superalgebras by noncommutative ones, but in that step the geometric intuition that we had retained in supergeometry with the functor of points is lost. We have then to rely on the algebraic counterpart of the geometric objects and try to generalize them to the non commutative setting. Non commutative geometry [4] is certainly the most complete framework to do so, and ultimately it will be connected to the quantization of space and superspace.

The approach that we follow here is the following: we substitute the supergroups by quantum supergroups and the corresponding homogeneous spaces by quantum homogeneous spaces. This was the approach followed in [7-9] for the nonsuper case. We are then able to preserve also the realization of the quantized super Minkowski space as the "big cell" (appropriately defined) of the quantum conformal space.

The problem of quantizing the Minkowski superspace has appeared in many places in the physics literature. We mention some references, although our list is not exhaustive. We find a first step as early as in [10,11], and in deformations (mostly with constant Poisson bracket) inspired in string theory $[12-15]$. In other papers, one finds the quantization of a super Minkowski "phase space", which, although it is not exactly the problem that we examine here, it is also of interest [16-18]. Quantizations for other superspaces such as supergroups [19-21], their coadjoint orbits [22] or other homogeneous superspaces $\mathbf{P}^{m \mid n}[23]$ are constructed.

The principle that guides us in choosing a particular deformation is that we want them to preserve the action of the corresponding symmetry groups and we also ask that the quantum Minkowski superspace appears as the big cell (appropriately defined in algebraic terms) inside the quantum conformal 
superspace. Obviously, all of these requests have to be made precise in the framework of deformation quantization.

The content of the present paper is as follows.

We start in Section 2 by giving an overview on the physical question that originates our discussion. We are after a rigorous mathematical description of the chiral superfields and their quantization, making sure to preserve the natural supergroup actions of the superconformal and super Poincaré groups.

We will devote Section 3 to give an intuitive explanation of how the functor of points works in terms of superalgebras.

In Section 4, we review briefly the classical theory of the Grassmannian manifold as embedded in the projective space. This is the standard Plücker embedding. We then generalize these structures to the super setting.

In Section 5, we use the technology developed in the series of papers [7-9], to quantize the Grassmannian and flag supervarieties by replacing the symmetry supergroup by a quantum supergroup. We then discuss quantum supergroups and their homogeneous spaces [1,19-21], by looking at the corresponding non commutative superalgebras. One result of this approach is that although the superalgebras become non commutative, the group law, represented by the comultiplication in the quantum supergroup, is not deformed. This can be interpreted by saying that the physical symmetry principle remains intact in the process of quantization.

In Section 6, we give a definition of the quantum big cell inside the quantum Grassmannian, presenting a coaction of the quantum super Poincaré group.

In Section 7, we state our conclusions and give the guidelines for further work.

We have left for Appendix A a brief and formal account of the most fundamental concepts of supergeometry.

\section{Notation.}

We will say that a superalgebra is commutative if for two elements $a$ and $b$ of definite parities $p_{a}$ and $p_{b}$ we have

$$
a \cdot b=(-1)^{p_{a} p_{b}} b \cdot a .
$$


In physics this is usually called an (anti)commutative or supercommutative algebra, but we prefer to keep the word commutative as in [5].

In this paper the word "classical" is used for commutative superalgebras, as opposed to the "quantum" superalgebras, which are noncommutative.

Also, we stress that all our definitions are done in terms of algebras and superalgebras, since it is the only aspect that survives in the noncommutative case.

\section{Real and chiral superfields in Minkowski superspace}

We want to devote this section to introduce real and chiral superfields as they are used in physics as well as to motivate the importance of having them quantized. For this purpose we will consider superfields in super Minkowski space: one can then introduce the notion of conformal or superconformal invariance in quantum field theory.

\subsection{Definitions}

We consider the complexified Minkowski space $\mathbb{C}^{4}$. The $N=1$ scalar superfields on the complexified Minkowski space are elements of the commutative superalgebra

$$
\mathcal{O}\left(\mathbb{C}^{4 \mid 4}\right) \equiv C^{\infty}\left(\mathbb{C}^{4}\right) \otimes \Lambda\left[\theta^{1}, \theta^{2}, \bar{\theta}^{1}, \bar{\theta}^{2}\right],
$$

where $\Lambda\left[\theta^{1}, \theta^{2}, \bar{\theta}^{1}, \bar{\theta}^{2}\right]$ is the Grassmann (or exterior) algebra generated by the odd variables $\theta^{1}, \theta^{2}, \bar{\theta}^{1}, \bar{\theta}^{2}$. Giving this superalgebra is equivalent to giving the superspace $\mathbb{C}^{4 \mid 4}$ as defined in Appendix A.1.

We will denote the coordinates (or generators) of the superspace as

$$
\begin{array}{cl}
x^{\mu}, & \mu=0,1,2,3 \quad \text { (even coordinates), } \\
\theta^{\alpha}, \bar{\theta}^{\dot{\alpha}}, & \alpha, \dot{\alpha}=1,2 \quad \text { (odd coordinates), }
\end{array}
$$

and a superfield, in terms of its field components, as

$$
\begin{aligned}
\Psi(x, \theta, \bar{\theta})= & \psi_{0}(x)+\psi_{\alpha}(x) \theta^{\alpha}+\psi_{\dot{\alpha}}^{\prime}(x) \bar{\theta}^{\dot{\alpha}}+\psi_{\alpha \beta}(x) \theta^{\alpha} \theta^{\beta}+\psi_{\alpha \dot{\beta}}(x) \theta^{\alpha} \bar{\theta}^{\dot{\beta}} \\
& +\psi_{\dot{\alpha} \dot{\beta}}^{\prime}(x) \bar{\theta}^{\dot{\alpha}} \bar{\theta}^{\dot{\beta}}+\psi_{\alpha \beta \dot{\gamma}}(x) \theta^{\alpha} \theta^{\beta} \bar{\theta}^{\dot{\gamma}}+\psi_{\alpha \dot{\beta} \dot{\gamma}}^{\prime}(x) \theta^{\alpha} \bar{\theta}^{\dot{\beta}} \bar{\theta}^{\dot{\gamma}} \\
& +\psi_{\alpha \beta \dot{\gamma} \dot{\delta}}(x) \theta^{\alpha} \theta^{\beta} \bar{\theta}^{\dot{\gamma}} \bar{\theta}^{\dot{\delta}} .
\end{aligned}
$$


A conjugation on a superalgebra $\mathcal{A}$ (not necessarily commutative) is an antilinear involutive map satisfying

$$
(f \cdot g)^{*}=(-1)^{p_{f} p_{g}} g^{*} \cdot f^{*}, \quad f, g \in \mathcal{A}
$$

where $p_{f}$ is the parity of the element $f$. Here we take the convention of [5] (see page 89 in there for a detailed explanation) because it has categorical meaning. Moreover, it is much more appropriate for superalgebras that are not necessarily commutative. It differs from the one used in physics, because if $f$ and $g$ are odd elements, then

$$
(f \cdot g)^{*}=-g^{*} \cdot f^{*},
$$

and if the superalgebra is commutative

$$
(f \cdot g)^{*}=f^{*} \cdot g^{*} .
$$

Instead, physicists use an operation such that

$$
(f \cdot g)^{\rho}=g^{\rho} \cdot f^{\rho},
$$

but this gives rise to minus signs and "i" factors that we would like to avoid. As explained in [5], one convention can be reverted into the other by a change

$$
f^{*}= \begin{cases}f^{\rho} & \text { if } f \text { is even } \\ \text { if } f^{\rho} & \text { if } f \text { is odd }\end{cases}
$$

so

$$
(f \cdot g)^{\rho}=g^{\rho} \cdot f^{\rho} .
$$

On the space of complex functions $C^{\infty}\left(\mathbb{C}^{4}\right)$, there exists the standard complex conjugation, denoted as

$$
f^{*}=\bar{f}, \quad f \in C^{\infty}\left(\mathbb{C}^{4}\right) .
$$

Hence, to give a conjugation it is enough to give it on the odd generators. This is done formally in the following way

$$
\left(\theta^{\alpha}\right)^{*}=\bar{\theta}^{\dot{\alpha}}, \quad\left(\bar{\theta}^{\dot{\alpha}}\right)^{*}=\theta^{\alpha} .
$$

This is then extended by (2.2) and antilinearity to the whole superalgebra $\mathcal{O}\left(\mathbb{C}^{4 \mid 4}\right)$. Real superfields then belong to $\mathcal{O}\left(\mathbb{R}^{4 \mid 4}\right)$. 
Using the convention (2.2) a superfield $\Psi(x, \theta, \bar{\theta})$ is real if and only if its field components satisfy

$$
\begin{aligned}
& \psi_{0}^{*}=\psi_{0}, \quad \psi_{\alpha}^{*}=\psi_{\dot{\alpha}}^{\prime}, \quad \psi_{\alpha \beta}^{*}=\psi_{\dot{\alpha} \dot{\beta}}^{\prime}, \quad \psi_{\alpha \dot{\beta}}^{*}=-\psi_{\beta \dot{\alpha}}, \\
& \psi_{\alpha \dot{\beta} \dot{\gamma}}^{\prime}=\psi_{\alpha \beta \dot{\gamma}}^{*}, \quad \psi_{\alpha \beta \dot{\gamma} \dot{\delta}}^{*}=\psi_{\gamma \delta \dot{\alpha} \dot{\beta}} .
\end{aligned}
$$

Action of the Lorentz group $\mathbf{S O}(1,3)$. There is an action of the double covering of the complexified Lorentz group, $\operatorname{Spin}(1,3)^{c} \approx \mathrm{SL}(2, \mathbb{C}) \times$ $\operatorname{SL}(2, \mathbb{C})$ over $\mathbb{C}^{4 \mid 4}$. The even coordinates $x^{\mu}$ transform in the fundamental representation of $\mathrm{SO}(1,3)(V)$,

$$
x^{\mu} \mapsto \Lambda_{\nu}^{\mu} x^{\nu},
$$

while $\theta$ and $\bar{\theta}$ are Weyl spinors (or half-spinors). More precisely, the coordinates $\theta$ transform in one of the spinor representations, say $S^{+} \approx(1 / 2,0)$, and $\bar{\theta}$ transform in the opposite chirality representation, $S^{-} \approx(0,1 / 2)$,

$$
\theta^{\alpha} \mapsto S^{\alpha}{ }_{\beta} \theta^{\beta}, \quad \bar{\theta}^{\dot{\alpha}} \mapsto \tilde{S}^{\dot{\alpha}} \bar{\theta}^{\dot{\beta}} .
$$

In fact, for the real form $\operatorname{Spin}(1,3)$, the representations $S^{+}$and $S^{-}$are complex, and they are related by complex conjugation, so this is consistent with the rule (2.3).

The scalar superfields are invariant under the action of the Lorentz group,

$$
\Psi(x, \theta, \bar{\theta})=(R \Psi)\left(\Lambda^{-1} x, S^{-1} \theta, \tilde{S}^{-1} \bar{\theta}\right),
$$

where $R \Psi$ is the superfield obtained by transforming the field components

$$
R \psi_{0}(x)=\psi_{0}(x), \quad R \psi_{\alpha}(x)=S_{\alpha}{ }^{\beta} \psi_{\beta}(x), \ldots
$$

The Hermitian matrices

$$
\sigma^{0}=\left(\begin{array}{ll}
1 & 0 \\
0 & 1
\end{array}\right), \quad \sigma^{1}=\left(\begin{array}{ll}
0 & 1 \\
1 & 0
\end{array}\right), \quad \sigma^{2}=\left(\begin{array}{cc}
0 & -\mathrm{i} \\
\mathrm{i} & 0
\end{array}\right), \quad \sigma^{3}=\left(\begin{array}{ll}
1 & 0 \\
0 & 1
\end{array}\right),
$$

define a $\operatorname{Spin}(1,3)$-morphism

$$
\begin{aligned}
S^{+} \otimes S^{-} & \longrightarrow V, \\
s^{\alpha} \otimes t^{\dot{\alpha}} & \longrightarrow s^{\alpha} \sigma_{\alpha \dot{\alpha}}^{\mu} t^{\dot{\alpha}} .
\end{aligned}
$$


Derivations. A left derivation of degree $m=0,1$ of a super algebra $\mathcal{A}$ is a linear map $D^{\mathrm{L}}: \mathcal{A} \mapsto \mathcal{A}$ such that

$$
D^{\mathrm{L}}(\Psi \cdot \Phi)=D^{\mathrm{L}}(\Psi) \cdot \Phi+(-1)^{m p_{\Psi}} \Psi \cdot D^{\mathrm{L}}(\Phi) .
$$

Graded left derivations span a $\mathbb{Z}_{2}$-graded vector space (or supervector space).

In general, linear maps over a supervector space are also a $\mathbb{Z}_{2}$-graded vector space. A map has degree 0 if it preserves the parity and degree 1 if it changes the parity. For the case of derivations of a commutative superalgebra, an even derivation has degree 0 as a linear map and an odd derivation has degree 1 as a linear map.

In the same way, one defines right derivations,

$$
D^{\mathrm{R}}(\Psi \cdot \Phi)=(-1)^{m p_{\Phi}} D^{\mathrm{R}}(\Psi) \cdot \Phi+\Psi \cdot D^{\mathrm{R}}(\Phi) .
$$

Note that derivations of degree zero are both, right and left derivations. Moreover, given a left derivation $D^{\mathrm{L}}$ of degree $m$, one can define a right derivation $D^{\mathrm{R}}$ also of degree $m$ in the following way

$$
D^{\mathrm{R}} \Psi=(-1)^{m\left(p_{\Psi}+1\right)} D^{\mathrm{L}} \Psi
$$

Let us now focus on the commutative superalgebra $\mathcal{O}\left(\mathbb{C}^{4 \mid 4}\right)$. We define the standard left derivations

$$
\begin{aligned}
& \partial_{\alpha}^{\mathrm{L}} \Psi=\psi_{\alpha}+2 \psi_{\alpha \beta} \theta^{\beta}+\psi_{\alpha \dot{\beta}} \bar{\theta}^{\dot{\beta}}+2 \psi_{\alpha \beta \dot{\gamma}} \theta^{\beta} \bar{\theta}^{\dot{\gamma}}+\psi_{\alpha \dot{\beta} \dot{\gamma}}^{\prime} \bar{\theta}^{\dot{\beta}} \bar{\theta}^{\dot{\gamma}}+2 \psi_{\alpha \beta \dot{\gamma} \dot{\delta}} \theta^{\beta} \bar{\theta}^{\dot{\gamma}} \bar{\theta}^{\dot{\delta}}, \\
& \partial_{\dot{\alpha}}^{\mathrm{L}} \Psi=\psi_{\dot{\alpha}}^{\prime}-\psi_{\beta \dot{\alpha}} \theta^{\beta}+2 \psi_{\dot{\alpha} \dot{\beta}}^{\prime} \bar{\theta}^{\dot{\beta}}+\psi_{\gamma \beta \dot{\alpha}} \theta^{\gamma} \theta^{\beta}-2 \psi_{\beta \dot{\alpha} \dot{\gamma}}^{\prime} \theta^{\beta} \bar{\theta}^{\dot{\gamma}}+2 \psi_{\gamma \beta \dot{\alpha} \dot{\delta}} \theta^{\gamma} \theta^{\beta} \bar{\theta}^{\dot{\delta}} .
\end{aligned}
$$

With our convention (2.2), one has that

$$
\left(\partial_{\alpha}^{\mathrm{L}} \Psi\right)^{*}=\partial_{\dot{\alpha}}^{\mathrm{L}} \Psi^{*}
$$

Also using (2.4), one can define $\partial_{\alpha}^{R}, \partial_{\dot{\alpha}}^{R}$. They have the same property than the left derivatives under complex conjugation.

We consider now the odd, left derivations

$$
Q_{\alpha}^{\mathrm{L}}=\partial_{\alpha}^{\mathrm{L}}-\mathrm{i} \sigma_{\alpha \dot{\alpha}}^{\mu} \bar{\theta}^{\dot{\alpha}} \partial_{\mu}, \quad \bar{Q}_{\dot{\alpha}}^{\mathrm{L}}=-\partial_{\dot{\alpha}}^{\mathrm{L}}+\mathrm{i} \theta^{\alpha} \sigma_{\alpha \dot{\alpha}}^{\mu} \partial_{\mu} .
$$

They satisfy the anticommutation rules

$$
\left\{Q_{\alpha}^{\mathrm{L}}, \bar{Q}_{\dot{\alpha}}^{\mathrm{L}}\right\}=2 \mathrm{i} \sigma_{\alpha \dot{\alpha}}^{\mu} \frac{\partial}{\partial x^{\mu}}, \quad\left\{Q_{\alpha}^{\mathrm{L}}, Q_{\beta}^{\mathrm{L}}\right\}=\left\{\bar{Q}_{\dot{\alpha}}^{\mathrm{L}}, \bar{Q}_{\dot{\beta}}^{\mathrm{L}}\right\}=0 .
$$


$Q^{\mathrm{L}}$ and $\bar{Q}^{\mathrm{L}}$ are the supersymmetry charges or supercharges. Together with

$$
P^{\mu}=-\mathrm{i} \frac{\partial}{\partial x^{\mu}},
$$

they form a Lie superalgebra, the supertranslation algebra, which then acts on the superspace $\mathbb{C}^{4 \mid 4}$.

Let us define another set of (left) derivations,

$$
D_{\alpha}^{\mathrm{L}}=\frac{\partial^{\mathrm{L}}}{\partial \theta^{\alpha}}+\mathrm{i} \sigma_{\alpha \dot{\alpha}}^{\mu} \bar{\theta}^{\dot{\alpha}} \frac{\partial}{\partial x^{\mu}}, \quad \bar{D}_{\dot{\alpha}}^{\mathrm{L}}=-\frac{\partial^{\mathrm{L}}}{\partial \bar{\theta}^{\dot{\alpha}}}-\mathrm{i} \theta^{\alpha} \sigma_{\alpha \dot{\alpha}}^{\mu} \frac{\partial}{\partial x^{\mu}},
$$

with anticommutation rules

$$
\left\{D_{\alpha}^{\mathrm{L}}, \bar{D}_{\dot{\alpha}}^{\mathrm{L}}\right\}=-2 \mathrm{i} \sigma_{\alpha \dot{\alpha}}^{\mu} \frac{\partial}{\partial x^{\mu}}, \quad\left\{D_{\alpha}^{\mathrm{L}}, D_{\beta}^{\mathrm{L}}\right\}=\left\{\bar{D}_{\dot{\alpha}}^{\mathrm{L}}, \bar{D}_{\dot{\beta}}^{\mathrm{L}}\right\}=0 .
$$

They also form a Lie superalgebra, isomorphic to the supertranslation algebra. This can be seen by taking

$$
Q^{\mathrm{L}} \rightarrow-D^{\mathrm{L}}, \quad \bar{Q}^{\mathrm{L}} \longrightarrow \bar{D}^{\mathrm{L}} .
$$

It is easy to see that the supercharges anticommute with the derivations $D^{\mathrm{L}}$ and $\bar{D}^{\mathrm{L}}$. For this reason, $D^{\mathrm{L}}$ and $\bar{D}^{\mathrm{L}}$ are called supersymmetric covariant derivatives or simply covariant derivatives, although they are not related to any connection form.

We go now to the central definition.

Definition 2.1. A chiral superfield is a superfield $\Phi$ such that

$$
\bar{D}_{\dot{\alpha}}^{\mathrm{L}} \Phi=0 .
$$

Because of the anticommuting properties of $D^{\prime} s$ and $Q^{\prime} s$, we have that

$$
\bar{D}_{\dot{\alpha}}^{\mathrm{L}} \Phi=0 \quad \Rightarrow \quad \bar{D}_{\dot{\alpha}}^{\mathrm{L}}\left(Q_{\beta}^{\mathrm{L}} \Phi\right)=0, \quad \bar{D}_{\dot{\alpha}}^{\mathrm{L}}\left(\bar{Q}_{\dot{\beta}}^{\mathrm{L}}\right) \Phi=0 .
$$

This means that the supertranslation algebra acts on the space of chiral superfields.

On the other hand, due to the derivation property,

$$
\bar{D}_{\dot{\alpha}}^{\mathrm{L}}(\Phi \Psi)=\bar{D}_{\dot{\alpha}}^{\mathrm{L}}(\Phi) \Psi+(-1)^{p_{\Phi}} \Phi \bar{D}_{\dot{\alpha}}^{\mathrm{L}}(\Psi),
$$

we have that the product of two chiral superfields is again a chiral superfield. 


\subsection{Shifted coordinates}

One can solve the constraint (2.5) in the following way. Note that the quantities

$$
y^{\mu}=x^{\mu}+i \theta^{\alpha} \sigma_{\alpha \dot{\alpha}}^{\mu} \bar{\theta}^{\dot{\alpha}}, \quad \theta^{\alpha}
$$

satisfy

$$
\bar{D}_{\dot{\alpha}}^{\mathrm{L}} y^{\mu}=0, \quad \bar{D}_{\dot{\alpha}}^{\mathrm{L}} \theta^{\alpha}=0,
$$

and using the derivation property, any superfield of the form

$$
\Phi\left(y^{\mu}, \theta\right), \quad \text { satisfies } \quad \bar{D}_{\dot{\alpha}}^{\mathrm{L}} \Phi=0
$$

and so it is a chiral superfield. This is the general solution of (2.5).

We can make the change of coordinates

$$
x^{\mu}, \theta^{\alpha}, \bar{\theta}^{\dot{\alpha}} \longrightarrow y^{\mu}=x^{\mu}+i \theta^{\alpha} \sigma_{\alpha \dot{\alpha}}^{\mu} \bar{\theta}^{\dot{\alpha}}, \theta^{\alpha}, \bar{\theta}^{\dot{\alpha}} .
$$

A superfield may be expressed in both coordinate systems

$$
\Phi(x, \theta, \bar{\theta})=\Phi^{\prime}(y, \theta, \bar{\theta}) .
$$

The covariant derivatives and supersymmetry charges take the form

$$
\begin{array}{rlrl}
D_{\alpha}^{\mathrm{L}} \Phi^{\prime} & =\frac{\partial^{\mathrm{L}} \Phi^{\prime}}{\partial \theta^{\alpha}}+2 i \sigma_{\alpha \dot{\alpha}}^{\mu} \bar{\theta}^{\dot{\alpha}} \frac{\partial^{\mathrm{L}} \Phi^{\prime}}{\partial y^{\mu}}, & \bar{D}_{\dot{\alpha}}^{\mathrm{L}} \Phi^{\prime}=-\frac{\partial^{\mathrm{L}} \Phi^{\prime}}{\partial \bar{\theta}^{\dot{\alpha}}}, \\
\bar{Q}_{\dot{\alpha}}^{\mathrm{L}} \Phi^{\prime}=-\frac{\partial^{\mathrm{L}} \Phi^{\prime}}{\partial \bar{\theta}^{\dot{\alpha}}}+2 i \theta^{\alpha} \sigma_{\alpha \dot{\alpha}}^{\mu} \frac{\partial^{\mathrm{L}} \Phi^{\prime}}{\partial y^{\mu}}, & Q_{\alpha}^{\mathrm{L}} \Phi^{\prime}=\frac{\partial^{\mathrm{L}} \Phi^{\prime}}{\partial \theta^{\alpha}} .
\end{array}
$$

In the new coordinate system, the chirality condition is simply

$$
\frac{\partial^{\mathrm{L}} \Phi^{\prime}}{\partial \bar{\theta}^{\dot{\alpha}}}=0
$$

so it is similar to a holomorphicity condition on the $\theta$ 's.

This shows that chiral scalar superfields are elements of the commutative superalgebra $\mathcal{O}\left(\mathbb{C}^{4 \mid 2}\right)=\mathbb{C}^{\infty}\left(\mathbb{C}^{4}\right) \otimes \Lambda\left[\theta^{1}, \theta^{2}\right]$. We shall realize this superspace as the big cell inside the chiral conformal superspace, which is the Grassmannian of $2 \mid 0$-subspaces of $\mathbb{C}^{4 \mid 1}$.

The complete (nonchiral) conformal superspace is in fact the flag supervariety of $2 \mid 0$-subspaces inside $2 \mid 1$-subspaces of $\mathbb{C}^{4 \mid 1}$. On this supervariety 
one can put a reality condition, and the real Minkowski superspace is the big cell inside the superflag. It is instructive to compare equation (2.6) with the incidence relation for the big cell of the flag manifold in equation (12) of [1]. We can then be convinced that the Grassmannian that we use to describe chiral superfields is inside the (complex) superflag.

\subsection{Supersymmetric theories}

Wess-Zumino models are supersymmetric models for one or several chiral superfields. These were the first type of supersymmetric theories that were written down [24]. Chiral superfields also appear in super Yang-Mills theories $[25,26]$, where the parameter of the gauge transformation is itself a chiral superfield.

The study of the chiral super Minkowski space is then justified from the physical point of view. Of course, most of the theories make use of the real super Minkowski space, and one needs also to consider real fields to formulate supersymmetric theories.

Also, it is important to consider the embedding of super Minkowski space inside conformal superspace, since some theories (for example, some WessZumino models and $N=4$ super Yang-Mills theory) have this symmetry. In fact, for the classic (nonquantum) case, this has been done in the modern language of supergeometry in [1].

In this paper though, we want to consider a quantization of these superspaces that preserves the action of the corresponding supergroups. It has been particularly difficult to find deformations of the space of chiral superfields involving also the odd variables $[13,15]$. Up to now, this has prevented to formulate Wess-Zumino or Yang-Mills models in a noncommutative superspace with a nontrivial deformation of the odd part and preserving the supersymmetry. Essentially, what happened in previous formulations is that the covariant derivatives were not anymore derivations of the noncommutative product, and then the ring of chiral superfields did not extend to a quantum chiral ring. Some proposals to keep a chiral ring (but not an antichiral one) include the partial (explicit) breaking of supersymmetry $[13,14]$.

In our formulation, we start with the classical chiral ring and find a quantum chiral ring in a natural way. We substitute the supergroup by a quantum supergroup and preserving the relations among all the elements of the construction. As it is well known, the comultiplication is not deformed when going from the classic to the quantum group, which means that the 
supersymmetry algebra is preserved without deformation although now it is realized on a noncommutative superspace. Mathematically, this is already a nontrivial problem, and physically it is a problem that must be solved in order to formulate the theories that use chiral superfields in noncommutative spaces.

Our approach will be complete once we extend it to the real super Minkowski space. In order to do this, one has to deal with the flag supermanifold. The Grassmannian then sits inside the complexified flag supermanifold.

One can certainly extend the same philosophy of quantization to the flag supervariety. Nevertheless, the problem is nontrivial, presents its own complications and will be the subject of a forthcoming paper.

Finally, since the superconformal symmetry is implicit in our approach, we expect to obtain in the future a basis to formulate conformal theories in a noncommutative space. This will include for example $N=4$ super Yang-Mills.

\section{The functor of points}

The functor of points is an extremely useful tool in classical algebraic geometry, which becomes essential in supergeometry in order to recover the geometric intuition, otherwise lost. In this section, we shall give an intuitive, though operative, summary of its definitions and properties, sending the reader to Appendix A for the complete treatment and all the references.

For definiteness, we take the ground field to be $k=\mathbb{R}, \mathbb{C}$. A superalgebra $\mathcal{A}$ is a $\mathbb{Z}_{2}$-graded algebra, $\mathcal{A}=\mathcal{A}_{0} \oplus \mathcal{A}_{1}$, where $p(x)$ denotes the parity of a homogeneous element $x$, so $p(x)=0$ if $x \in \mathcal{A}_{0}$ and $p(x)=1$ if $x \in \mathcal{A}_{1}$. The subspace $\mathcal{A}_{0}$ is an algebra, while the subspace $\mathcal{A}_{1}$ is an $\mathcal{A}_{0}$-module.

The superalgebra $\mathcal{A}$ is said to be commutative if for any two homogeneous elements $x, y$

$$
x y=(-1)^{p(x) p(y)} y x .
$$

From now on all superalgebras are assumed to be commutative unless otherwise specified. The category of commutative superalgebras will be denoted by (salg), and the category of sets is denoted by (sets). 
A functor $h:($ salg $) \longrightarrow$ (sets) is representable if there exists a superalgebra $\mathcal{B}$ such that

$$
\begin{aligned}
h:(\mathrm{salg}) & \longrightarrow \\
\mathcal{A} & \longrightarrow h(\mathcal{A})=\operatorname{Hom}(\mathcal{B}, \mathcal{A}) .
\end{aligned}
$$

We say that the superalgebra representing the functor $h$ is $\mathcal{B}$. In that case, we will denote the functor $h$ as $h_{\mathcal{B}}$, to stress the fact that it is represented by the superalgebra $\mathcal{B}$. An element of $\operatorname{Hom}(\mathcal{B}, \mathcal{A})$ is called an $\mathcal{A}$-point of $h_{\mathcal{B}}$.

If we restrict the functor $h$ to the category of $k$-algebras (that is, if we remove the odd generators), and we also demand that the representing algebras are reduced ${ }^{1}$ and finitely generated (these algebras are often called affine algebras), then a representable functor $h$ corresponds to an algebra $\mathcal{O}(X)$, that is the coordinate ring of an affine variety $X$.

Example 3.1. Let $S^{2}$ be the unit 2 -sphere in $\mathbb{C}^{3}$. Its coordinate ring is given by

$$
\mathcal{O}\left(S^{2}\right)=\mathbb{C}[x, y, z] /\left(x^{2}+y^{2}+z^{2}-1\right) .
$$

The elements in $\mathcal{O}\left(S^{2}\right)$ correspond to the polynomial functions on the variety $S^{2} \subset \mathbb{C}^{3}$. Each morphism $\mathcal{O}\left(S^{2}\right) \rightarrow \mathbb{C}$ is given in terms of the images of the generators

$$
(x, y, z) \longrightarrow(a, b, c) \text { with } a, b, c \in \mathbb{C} \text { such that } a^{2}+b^{2}+c^{2}=1,
$$

hence, any such morphism represents a point of the sphere. If we consider morphisms $\mathcal{O}\left(S^{2}\right) \rightarrow \mathbf{Q}$, they are in one-to-one correspondence with rational points on the sphere. In this example, the geometric points (that is the $\mathbb{C}$ points) allow us to recover all the information on $S^{2}$.

In Appendix A, we give the definition of affine supervariety; however, for all the practical purposes in this paper, we can identify an affine supervariety $X$ with its coordinate ring $\mathcal{O}(X)$, this time $\mathcal{O}(X)$ being a superalgebra. As in the nonsuper case, it results that affine supervarieties are in one to one correspondence with affine superalgebras. This means only that the ordinary algebra obtained by taking modulo by the odd ideal $\mathcal{O}(X) / \mathcal{I}_{\text {odd }}$ is an affine algebra. Then, there is an affine variety corresponding to $\mathcal{O}(X) / \mathcal{I}_{\text {odd }}$. This is an affine variety underlying the affine supervariety.

\footnotetext{
${ }^{1} \mathrm{An}$ algebra is reduced if it has no nilpotent elements.
} 
The $\mathcal{A}$-points of the supervariety $X$ are the morphisms $\mathcal{O}(X) \longrightarrow \mathcal{A}$ and all the information about the affine supervariety is fully encoded in its coordinate superalgebra, or equivalently in its functor of points

$$
\begin{aligned}
h_{X}:(\mathrm{salg}) & \longrightarrow \\
\mathcal{A} & \longrightarrow h_{X}(\mathcal{A})=\operatorname{Hom}(\mathcal{O}(X), \mathcal{A}) .
\end{aligned}
$$

We have denoted $h_{X}$ instead of $h_{\mathcal{O}(X)}$ for simplicity, since there is no possibility of confusion in this context.

To clarify these concepts we describe a simple example.

Example 3.2. We define the polynomial superalgebra as:

$$
k\left[x^{1}, \ldots, x^{p}, \theta^{1}, \ldots, \theta^{q}\right]:=k\left[x^{1}, \ldots, x^{p}\right] \otimes \Lambda\left[\theta^{1}, \ldots, \theta^{q}\right] .
$$

We want to interpret this superalgebra as the coordinate ring of the affine superspace of superdimension $p \mid q$, that we shall denote with the symbol $k^{p \mid q}$. If $\mathcal{A}$ is a generic (commutative) superalgebra, an $\mathcal{A}$-point of $k^{p \mid q}$ is given by a morphism $k\left[x^{1}, \ldots, x^{p}, \theta^{1}, \ldots, \theta^{q}\right] \longrightarrow \mathcal{A}$, which is determined once we know the images of the generators

$$
\left(x^{1}, \ldots, x^{p}, \theta^{1}, \ldots, \theta^{q}\right) \longrightarrow\left(a^{1} \ldots a^{p}, \alpha^{1} \ldots \alpha^{q}\right),
$$

with $a^{i} \in \mathcal{A}_{0}$ and $\alpha^{j} \in \mathcal{A}_{1}$. Note that the $k$-points of $k^{p \mid q}$ are given by $\left(k_{1} \ldots k_{p}, 0 \ldots 0\right)$ and coincide with the points of the affine space $k^{p}$. From this example it is clear that the knowledge of the geometric points, that is the $k$-points, is by no means sufficient to describe the supergeometric object.

There is an important property of the functor of points, which is constantly used and makes all calculations easier. In complete analogy with the classical setting much of the behaviour of the functor of points of an affine supervariety is captured by its image on the local superalgebras ${ }^{2}$ (For this fact see [6] Ch. 10). Local superalgebras are important, since their behavior resembles those of fields, thus rendering the functor of points easier to describe in the examples that we are interested in.

We now turn to projective supervarieties. In the classical setting projective varieties are harder to describe, since the homogeneous coordinate ring that we associate to a projective variety encodes not only the structure of the variety, but also its embedding into projective space. Such ring hence

\footnotetext{
${ }^{2} \mathrm{~A}$ local superalgebra is a superalgebra that has a unique, maximal ideal which is homogeneous with respect to the $\mathbb{Z}_{2}$ grading.
} 
has more information than the variety itself. As a consequence we have that nonisomorphic coordinate homogeneous rings may correspond to the same projective variety, a phenomenon that we do not see in the affine case, where coordinate rings and affine varieties correspond bijectively to each other and contain essentially the same information. A fancy way to express this, is to say that the category of affine (super)varieties over $k$ is equivalent to the category of affine $k$-(super)algebras. So in the affine setting we have that in both, the classical and the super setting,

$$
\operatorname{Hom}(\mathcal{O}(X), \mathcal{O}(Y))=\operatorname{Hom}(Y, X) .
$$

Note that the role of $X$ and $Y$ are interchanged when passing from the coordinate (super)rings to the (super)varieties.

There are two equivalent, but different, ways to approach projective varieties in the ordinary setting and we shall briefly describe them. Both can be generalized to the super setting. For us the second one is far more important, since it gives a setting suitable for the quantization. The first one will also be used (implicitly) when we perform the quantization of the big cell in Section 6.

One way to approach projective varieties is to view them locally as affine varieties that can suitably be patched together. In other words a projective variety is a topological space that is covered by affine varieties, whose coordinates, in the overlaps of different affine varieties, behave in a certain way. We can build the functor of points of a projective variety by giving the functors of points of the affine varieties and then asking that they satisfy certain gluing conditions. This is in essence the meaning of the Representability Theorem $^{3}$ in Appendix A.

A second equivalent way to define a projective variety $X$ is to look at the points in a projective space $\mathbf{P}^{n}$, satisfying homogeneous equations $f_{1}=0, \ldots, f_{n}=0$ (homogeneous here refers to the $\mathbb{Z}$-grading of polynomials). Hence the homogeneous ring $S=k\left[x_{0} \ldots x_{n}\right] /\left(f_{1}, \ldots, f_{n}\right)$ determines uniquely the projective variety $X$ together with its embedding into $\mathbf{P}^{n}$. However, as we have already remarked, the variety $X$ does not determine uniquely the ring $S$ : there could in fact be a different embedding of $X$ into some other projective space, yielding a homogeneous algebra nonisomorphic to $S$. For this reason the functor of points of a projective variety is more tricky and it is not directly related to the coordinate ring $S$ of the variety itself as it happens for the affine case. In particular this functor is

\footnotetext{
${ }^{3}$ The Representability Theorem is stated in the more general setting of superschemes, which are a far reaching generalization of projective supervarieties.
} 
never representable, hence making the theory more difficult since there is no algebra that can be naturally associated to it. Hence we are facing a new problem: how can we decide whether a functor $h:(\mathrm{salg}) \longrightarrow$ (sets) is the functor of points of a projective supervariety? Certainly we cannot say as before that this is equivalent for $h$ to be representable. The answer is again the Representability Theorem in Appendix A.

As an example of its application, let us examine the functor of points of the projective space $\mathbf{P}^{n}$.

Example 3.3. Let us consider the functor: $h:(\operatorname{alg}) \longrightarrow($ sets $)$, where $h(\mathcal{A})$ are the projective $\mathcal{A}$-modules of rank one in $\mathcal{A}^{n+1}$

Equivalently $h(\mathcal{A})$ consists of the pairs $(L, \phi)$, where $L$ is a projective $\mathcal{A}$ module of rank one, and $\phi$ is a surjective morphisms $\phi: \mathcal{A}^{n+1} \longrightarrow L$. These pairs are taken modulo the equivalence relation

$$
(L, \phi) \approx\left(L^{\prime}, \phi^{\prime}\right) \quad \Leftrightarrow \quad L \approx L^{\prime}, \quad \phi^{\prime}=a \circ \phi,
$$

where $a: L \rightarrow L^{\prime}$ is the isomorphism. We can substitute the last condition by saying that $\phi$ and $\phi^{\prime}$ have the same kernel.

If $\mathcal{A}=k$ is a field, then projective modules are free and a morphism

$$
\phi: k^{n+1} \rightarrow k
$$

is specified by a $n$-uple $\left(a^{1}, \ldots, a^{n+1}\right)$, with $a^{i} \in k$, not all of the $a^{i}=0$. The equivalence relation becomes

$$
\left(a^{1}, \ldots, a^{n+1}\right) \sim\left(b^{1}, \ldots, b^{n+1}\right) \quad \Leftrightarrow \quad\left(a^{1}, \ldots, a^{n+1}\right)=\lambda\left(b^{1}, \ldots, b^{n+1}\right),
$$

with $\lambda \in k^{\times}$understood as an automorphism of $k$. It is clear then that $h(k)$ consists of all the lines through the origin in the vector space $k^{n+1}$.

If $\mathcal{A}$ is local, projective modules are free over local rings. We then have a situation similar to the field setting: equivalence classes are lines in the $\mathcal{A}$-module $\mathcal{A}^{n+1}$. The functor $h$ is "covered" by functors which on a local algebra $\mathcal{A}$

$$
h_{i}(\mathcal{A})=\left\{\operatorname{span}\left\{\left(a^{1}, \ldots, a^{n+1}\right)\right\} \in \mathcal{A}^{n+1} \mid a^{i} \text { is invertible }\right\} .
$$

In fact, in order to correspond to a surjective morphism, the $n$-uple $\left(a^{1}, \ldots\right.$, $\left.a^{n+1}\right)$ must contain at least an invertible $a^{i}$. Hence, using the equivalence relation, we have that $h_{i}(\mathcal{A})=\left\{\left(a_{1} / a_{i}, \ldots, \hat{a}_{i}, \ldots a^{n+1} / a_{i}\right)\right\}$ and consequently $h_{i}=h_{\mathbf{A}^{n}}$, as described in Example 3.2. 
Using the Representability Theorem one can then show that the functor $h$ is the functor of points of a variety that we call the projective space and whose geometric points coincide with the projective space $\mathbf{P}^{n}$ over the field $k$ as we usually understand it.

This example can be easily generalized to the supercontext: we consider the functor $h_{\mathbf{P}^{m \mid n}}:$ (salg) $\longrightarrow$ (sets), where $h_{\mathbf{P}^{m \mid n}}(\mathcal{A})$ is defined as the set the projective $\mathcal{A}$-modules of rank $1 \mid 0$ in $\mathcal{A}^{m \mid n}:=\mathcal{A} \otimes k^{m \mid n}$. This is the functor of points of a supervariety, called the projective superspace.

The next question that we want to tackle is how we can define an embedding of a (super)variety into the projective (super)space using the functor of points notation.

Let $X$ be a projective supervariety and let $\Phi: X \longrightarrow \mathbf{P}^{n \mid m}$ be an injective morphism. In the notation of the functor of points, $\Phi$ is a natural transformation between the two functors $h_{X}$ and $h_{\mathbf{P}^{n \mid m}}$, given by

$$
\Phi_{\mathcal{A}}: h_{X}(\mathcal{A}) \longrightarrow h_{\mathbf{P}^{n \mid m}}(\mathcal{A})
$$

with $\Phi_{\mathcal{A}}$ injective.

If $\mathcal{A}$ is a local superalgebra, then an $\mathcal{A}$-point in $h_{\mathbf{P}^{n \mid m}(\mathcal{A})}$ is in $\Phi_{\mathcal{A}}\left(h_{X}(\mathcal{A})\right)$ if and only if it satisfies certain homogeneous polynomial relations $f_{1}=$ $0, \ldots, f_{r}=0$ in the indeterminates $x_{1}, \ldots, x_{n}, \xi_{1}, \ldots, \xi_{m}$. Moreover, $X$ is the projective supervariety associated to $k\left[x_{1}, \ldots, x_{n}, \xi_{1}, \ldots, \xi_{m}\right] /\left(f_{1}, \ldots, f_{r}\right)$. In other words the superalgebra $k\left[x_{1}, \ldots x_{n}, \xi_{1} \ldots, \xi_{m}\right] /\left(f_{1}, \ldots, f_{r}\right)$ is the coordinate superalgebra of the projective super variety $X$ with respect to the embedding $\Phi$. Recall that, although $X$ does not determine a coordinate superalgebra as in the affine case, the coordinate superalgebra of $X$ with respect to an embedding into projective superspace does determine the projective supervariety $X$.

In summary, to determine the coordinate superalgebra of a projective supervariety with respect to a certain projective embedding, we need to check the relations satisfied by the coordinates just on local superalgebras. This will be our starting point in Section 4.2 when we shall determine the coordinate superalgebra of the Grassmannian supervariety with respect to its Plücker embedding.

As a side remark we want to notice that the Grassmannian supervariety is not in general a projective supervariety $[27,28]$, contrary to the classical setting. This unpleasant feature luckily does not affect us, since the particular Grassmannian supervariety that we see in this paper is projective. 


\section{The super Grassmannian variety}

We will start by reviewing the Plücker embedding of the Grassmannian variety. We then turn to a description of the same object as a quotient space for the action of the special linear supergroup and we give an explicit description of the big cell inside the Grassmannian supervariety. The big cell is especially important since it is identified with the complex Minkowski superspace, while the subgroup of $\mathrm{SL}(4 \mid 1)$ stabilizing the big cell contains the Poincaré supergroup times the dilations.

Our point of view is purely algebraic, since it is the most suitable for the quantization. The reader however must be aware that these objects have a natural differential structure, as always is the case for smooth supergroups and the supervarieties on which they are acting [29].

\subsection{Plücker embedding of the Grassmannian variety and the big cell}

The Grassmannian variety $G(2,4)$ is the set of 2-planes inside a four dimensional space $\mathbb{C}^{4}$. It is a projective variety embedded in $\mathbf{P}^{5}$ and the embedding is known as the Plücker embedding. This can be found explained in many references (see for example $[1,3]$ ).

Let $\left\{e_{1}, e_{2}, e_{3}, e_{4}\right\}$ be the canonical basis of $\mathbb{C}^{4}$ and $\pi_{0}=\operatorname{span}\left\{e_{1}, e_{2}\right\}$ the 2-plane generated by $e_{1}$ and $e_{2}$. Then

$$
G(2,4)=\mathrm{SL}(4, \mathbb{C}) / P_{0},
$$

where the isotropy group $P_{0}$ is the subgroup that leaves invariant $\pi_{0}$ :

$$
P_{0}=\left\{\left(\begin{array}{cc}
L & M \\
0 & R
\end{array}\right) \in \mathrm{SL}(4, \mathbb{C})\right\}
$$

with $L, M, R$ being $2 \times 2$ matrices, and $L$ and $R$ invertible.

We will call $\mathrm{SL}(4, \mathbb{C})$ the conformal group in four dimensions. Indeed, this is the complexified spin group of $\mathrm{SO}(2,4)$, easily recognizable as the conformal group of the Minkowski metric in four dimensions, $\operatorname{diag}(1,-1,-1,-1)$. Note also that we can substitute $\mathrm{SL}(4, \mathbb{C})$ by $\mathrm{GL}(4, \mathbb{C})$ and the construction will also work.

To obtain the Plücker map, one starts by considering the vector space $E=$ $\Lambda^{2}\left(\mathbb{C}^{4}\right) \approx \mathbb{C}^{6}$ with basis $\left\{e_{i} \wedge e_{j}\right\}_{i<j}, i, j=1, \ldots, 4$. Then, for any plane 
$\pi=\operatorname{span}\{a, b\}$ we have

$$
a \wedge b=\sum_{i<j} y_{i j} e_{i} \wedge e_{j}
$$

A change of basis $\left(a^{\prime}, b^{\prime}\right)=(a, b) u$, where $u \in \mathrm{GL}(2, \mathbb{C})$, produces a change

$$
a^{\prime} \wedge b^{\prime}=\operatorname{det}(u) a \wedge b,
$$

so the image $\pi \rightarrow[a \wedge b]$ is well defined into the projective space $\mathbf{P}(E) \approx$ $\mathbf{P}^{5}$. This is called the Plücker map and it is not hard to see that it is an embedding. The quantities $y_{i j}$ are the homogeneous Plücker coordinates, and the image of the Plücker map can be identified with the solution of the equation $p \wedge p=0$ for $p \in E$. In coordinates, this reads

$$
y_{12} y_{34}+y_{23} y_{14}+y_{31} y_{24}=0, \quad\left(\text { with } y_{i j}=-y_{j i}\right) .
$$

Equation (4.1) is called the Plücker relation or Klein quadric. In algebraic terms, the fact that the Grassmannian is embedded in the projective space is reflected in the fact that the ideal $\mathcal{I}_{\mathrm{P}}$ generated by the relation (4.1) in $\operatorname{Pol}\left(\mathbb{C}^{6}\right)$ is homogeneous.

The Poincaré group plus dilatations and the big cell. We give an open cover for the projective variety $G(2,4)$. Let $a$ and $b$ be two linearly independent vectors spanning the 2-plane $\pi$ that can be represented by the matrix

$$
\pi=\operatorname{span}\{a, b\}=\left(\begin{array}{ll}
a_{1} & b_{1} \\
a_{2} & b_{2} \\
a_{3} & b_{3} \\
a_{4} & b_{4}
\end{array}\right) .
$$

This matrix has rank 2, so at least one of the 6 minors $y_{i j}=a_{i} b_{j}-b_{i} a_{j}$, $i<j$ is different from zero. The sets

$$
U_{i j}=\left\{(a, b) / y_{i j} \neq 0\right\}
$$

are open affine sets of $G(2,4)$ and cover it. $U_{12}$ is called the big cell. By a change of basis (action of $u \in \mathrm{GL}(2, \mathbb{C})$ ), a plane $\pi$ in the big cell can always be represented by the matrix

$$
\pi=\left(\begin{array}{l}
\mathbb{1 1} \\
A
\end{array}\right), \quad A=\left(\begin{array}{ll}
a_{11} & a_{12} \\
a_{21} & a_{22}
\end{array}\right),
$$

so $U_{12} \approx M_{2}(\mathbb{C}) \approx \mathbb{C}^{4}$, and $U_{12}$ is again the affine space $\mathbb{C}^{4}$, represented with the algebra $\mathbb{C}\left[a_{i j}\right]$. 
Given an element of $\mathrm{SL}(4, \mathbb{C})$, since the columns are linearly independent, we can choose the first two columns to be the vectors $a$ and $b$ representing a 2-plane $\pi$. If the plane is in the big cell, it is easy to see that there is a transformation in $P_{0}$ that brings the matrix of $\mathrm{SL}(4, \mathbb{C})$ to the form

$$
\left(\begin{array}{cc}
\mathbb{1}_{2} & 0 \\
A & 1_{2}
\end{array}\right)
$$

The big cell is left invariant by the subgroup of $\operatorname{SL}(4, \mathbb{C})$ consisting of the matrices of the form

$$
P=\left\{\left(\begin{array}{cc}
L & 0 \\
N L & R
\end{array}\right), L, R \text { invertible, } \operatorname{det} R \cdot \operatorname{det} L=1\right\}
$$

The bottom left entry is arbitrary but we have written it like that for convenience. The action on $U_{12}$ is then

$$
A \mapsto N+R A L^{-1},
$$

so $P$ has the structure of semidirect product $P=H \ltimes M_{2}$, where $M_{2}=\{N\}$ is the set of $2 \times 2$ matrices acting as translations, and

$$
H=\left\{\left(\begin{array}{ll}
L & 0 \\
0 & R
\end{array}\right), \quad L, R \in \mathrm{GL}(2, \mathbb{C}), \operatorname{det} L \cdot \operatorname{det} R=1\right\} .
$$

The subgroup $H$ is the direct product $\mathrm{SL}(2, \mathbb{C}) \times \mathrm{SL}(2, \mathbb{C}) \times \mathbb{C}^{\times}$. But $\mathrm{SL}(2, \mathbb{C}) \times \mathrm{SL}(2, \mathbb{C})$ is the spin group of $\mathrm{SO}(4, \mathbb{C})$, the complexified Lorentz group, and $\mathbb{C}^{\times}$acts as a dilation. $P$ is then the Poincaré group times dilations. In the basis of the Pauli matrices

$$
\sigma_{0}=\left(\begin{array}{cc}
1 & 0 \\
0 & 1
\end{array}\right), \quad \sigma_{1}=\left(\begin{array}{cc}
0 & 1 \\
1 & 0
\end{array}\right), \quad \sigma_{2}=\left(\begin{array}{cc}
0 & -\mathrm{i} \\
\mathrm{i} & 0
\end{array}\right), \quad \sigma_{3}=\left(\begin{array}{cc}
1 & 0 \\
0 & -1
\end{array}\right)
$$

an arbitrary matrix $A$ can be written as

$$
A=\left(\begin{array}{ll}
a_{11} & a_{12} \\
a_{21} & a_{22}
\end{array}\right)=x^{0} \sigma_{0}+x^{1} \sigma_{1}+x^{2} \sigma_{2}+x^{3} \sigma_{3}=\left(\begin{array}{cc}
x^{0}+x^{3} & x^{1}-\mathrm{i} x^{2} \\
x^{1}+\mathrm{i} x^{2} & x^{0}-x^{3}
\end{array}\right),
$$

and $\left(x^{0}, x^{1}, x^{2}, x^{3}\right)$ are the ordinary coordinates of Minkowski space. Moreover,

$$
\operatorname{det} A=\left(x^{0}\right)^{2}-\left(x^{1}\right)^{2}-\left(x^{2}\right)^{2}-\left(x^{3}\right)^{2} .
$$


This concludes the interpretation of the big cell as the complexification of the Minkowski space.

\subsection{The Plücker embedding for the super Grassmannian}

We are interested in the super Grassmannian of $(2 \mid 0)$ planes inside the superspace $\mathbb{C}^{4 \mid 1}$. Since we are only concerned with this particular Grassmannian, we will just denote it as $\mathrm{Gr}=G(2|0 ; 4| 1)$. In Appendix A we give an exhaustive description of its functor of points, $h_{\mathrm{Gr}}$. Here, we will use the fact that we can work on local algebras. Then the projective modules are free modules and the description is greatly simplified. On a local algebra $\mathcal{A}, h_{\mathrm{Gr}}(\mathcal{A})$ consists of free submodules of rank $2 \mid 0$ in $\mathcal{A}^{4 \mid 1}$. One such module can be specified by a couple of independent even vectors, which in the canonical basis $\left\{e_{1}, e_{2}, e_{3}, e_{4}, \mathcal{E}_{5}\right\}$ are

$$
\pi=\operatorname{span}\{a, b\}=\operatorname{span}\left\{\left(\begin{array}{ll}
a_{1} & b_{1} \\
a_{2} & b_{2} \\
a_{3} & b_{3} \\
a_{4} & b_{4} \\
\alpha_{5} & \beta_{5}
\end{array}\right)\right\},
$$

with $a_{i}, b_{i} \in \mathcal{A}_{0}$ and $\alpha_{5}, \beta_{5} \in \mathcal{A}_{1}$.

We want now to work out the expression for the Plücker embedding. We want to give a natural transformation among the functors

$$
p: h_{\mathrm{Gr}} \rightarrow h_{\mathbf{P}(E)} .
$$

where $E$ is the super vector space $E=\wedge^{2} \mathbb{C}^{4 \mid 1} \approx \mathbb{C}^{7 \mid 4}$. We recall that for an arbitrary super vector space $V$,

$$
\Lambda^{2} V=V \otimes V /\left\langle u \otimes v+(-1)^{|u||v|} v \otimes u\right\rangle, \quad u, v \in V .
$$

Given the canonical basis for $\mathbb{C}^{4 \mid 1}$ we construct a basis for $E$

$$
\begin{aligned}
e_{1} \wedge e_{2}, e_{1} \wedge e_{3}, e_{1} \wedge e_{4}, e_{2} \wedge e_{3}, e_{2} \wedge e_{4}, e_{3} \wedge e_{4}, \mathcal{E}_{5} \wedge \mathcal{E}_{5} \quad \text { (even) }, \\
e_{1} \wedge \mathcal{E}_{5}, e_{2} \wedge \mathcal{E}_{5}, e_{3} \wedge \mathcal{E}_{5}, e_{4} \wedge \mathcal{E}_{5} \quad \text { (odd) }
\end{aligned}
$$

As in the super vector space case, if $L$ is a $\mathcal{A}$-module, we can construct $\wedge^{2} L$

$$
\Lambda^{2} L=L \otimes L /\left\langle u \otimes v+(-1)^{|u||v|} v \otimes u\right\rangle, \quad u, v \in L .
$$

If $L \in h_{\mathrm{Gr}}(\mathcal{A})$, then $\wedge^{2} L \subset \wedge^{2} \mathcal{A}^{4 \mid 1}$. It is clear that if $L$ is a projective $\mathcal{A}$ module of rank $2 \mid 0$, then $\wedge^{2} L$ is a projective $\mathcal{A}$-module of rank $1 \mid 0$. In other 
words, it is an element of $h_{\mathbf{P}(E)}(\mathcal{A})$ for $E=\wedge^{2} \mathbb{C}^{4 \mid 1}$. Hence, we have defined a natural transformation:

$$
\begin{aligned}
h_{\mathrm{Gr}}(\mathcal{A}) \stackrel{p_{\mathcal{A}}}{\longrightarrow} & h_{\mathbf{P}(E)}(\mathcal{A}), \quad \mathcal{A} \in(\mathrm{salg}) . \\
L & \longrightarrow \wedge^{2} L,
\end{aligned}
$$

Once we have the natural transformation defined, we can restrict to work on local algebras. For a local algebra $\mathcal{A}, \operatorname{Gr}(\mathcal{A})$ consists of the free $2 \mid 0$-modules inside $\mathcal{A}^{4 \mid 1}$.

Let $a, b$ be two even independent vectors in $\mathcal{A}^{4 \mid 1}$. For any superalgebra $\mathcal{A}$, they generate a free submodule of $\mathcal{A}^{4 \mid 1}$ of rank $2 \mid 0$. The natural transformation described above is as follows.

$$
\begin{array}{cc}
h_{\mathrm{Gr}}(A) \stackrel{p_{\mathcal{A}}}{\longrightarrow} h_{\mathbf{P}(E)}(\mathcal{A}), \\
\operatorname{span}_{\mathcal{A}}\{a, b\} \longrightarrow \operatorname{span}_{\mathcal{A}}\{a \wedge b\},
\end{array}
$$

where $E=\Lambda^{2}\left(\mathbb{C}^{4 \mid 1}\right)$. The map $p_{\mathcal{A}}$ is clearly injective. The image $p_{\mathcal{A}}\left(h_{\mathrm{Gr}}(\mathcal{A})\right)$ is the subset of even elements in $h_{\mathbf{P}(E)}(\mathcal{A})$ decomposable in terms of two even vectors of $\mathcal{A}^{4 \mid 1}$. We are going to find the necessary and sufficient conditions for an even element $Q \in h_{\mathbf{P}(E)}(\mathcal{A})$ to be decomposable in terms of even vectors. In terms of the canonical basis (4.7) we have

$$
\begin{aligned}
& Q=q+\lambda \wedge \mathcal{E}_{5}+a_{55} \mathcal{E}_{5} \wedge \mathcal{E}_{5}, \quad \text { with } \\
& q=q_{12} e_{1} \wedge e_{2}+\cdots+q_{34} e_{3} \wedge e_{4}, \quad q_{i j} \in \mathcal{A}_{0}, \\
& \lambda=\lambda_{1} e_{1}+\cdots+\lambda_{4} e_{4}, \quad \lambda_{i} \in \mathcal{A}_{1} .
\end{aligned}
$$

$Q$ is decomposable if and only if

$$
\begin{aligned}
& Q=\left(r+\xi \mathcal{E}_{5}\right) \wedge\left(s+\theta \mathcal{E}_{5}\right) \quad \text { with } \\
& r=r_{1} e_{1}+\cdots+r_{4} e_{4}, \quad s=s_{1} e_{1}+\cdots+s_{4} e_{4}, \quad r_{i}, s_{i} \in \mathcal{A}_{0} \quad \xi, \theta \in \mathcal{A}_{1},
\end{aligned}
$$

which means

$$
Q=r \wedge s+(\theta r-\xi s) \wedge \mathcal{E}_{5}+\xi \theta \mathcal{E}_{5} \wedge \mathcal{E}_{5} .
$$

This is equivalent to

$$
q=r \wedge s, \quad \lambda=\theta r-\xi s, \quad a_{55}=\xi \theta,
$$

and these are in turn equivalent to the following:

$$
q \wedge q=0, \quad q \wedge \lambda=0, \quad \lambda \wedge \lambda=2 a_{55} q \quad \lambda a_{55}=0 .
$$


Plugging (4.8) we obtain

$$
\begin{array}{ll}
q_{12} q_{34}-q_{13} q_{24}+q_{14} q_{23}=0 & \text { (classical Plücker relation) } \\
q_{i j} \lambda_{k}-q_{i k} \lambda_{j}+q_{j k} \lambda_{i}=0, & 1 \leq i<j<k \leq 4 \\
\lambda_{i} \lambda_{j}=a_{55} q_{i j}, & 1 \leq i<j \leq 4 \\
\lambda_{i} a_{55}=0 . &
\end{array}
$$

These are the super Plücker relations. As we shall see in the next section the superalgebra

$$
\mathcal{O}(\mathrm{Gr})=k\left[q_{i j}, \lambda_{k}, a_{55}\right] / \mathcal{I}_{\mathrm{P}},
$$

is associated to the supervariety $\mathrm{Gr}$ in the Plücker embedding described above, where $\mathcal{I}_{\mathrm{P}}$ denotes the ideal of the super Plücker relations (4.9).

Remark 4.1. We want to stress that although $\mathcal{O}(\mathrm{Gr})$ does not represent the functor of points $h_{\mathrm{Gr}}$, (it is not representable as a functor from (salg) to (sets)), one can recover all the information about the supervariety from $\mathcal{O}(\mathrm{Gr})$ by the procedure described in Appendix A.2. (In the notation used there one has that $\mathcal{O}(\mathrm{Gr})=R$ ).

\subsection{The superstraightening algorithm}

We want to take a small digression to explain why the relations (4.9) generate the ideal $\mathcal{I}_{\mathrm{P}}$ of all the relations among the coordinates $q_{i j}, \lambda_{k}, a_{55}$. This could be done with a direct calculation as in [33], however we prefer to justify it by resorting to the theory of semistandard tableaux and the superstraightening algorithm. The algorithm has interest on his own, and we will also need these notions later, in the quantum setting.

Definition 4.1. A Young diagram is a finite collection of boxes, or cells, arranged in left-justified rows, with the row lengths weakly decreasing (each row has the same or shorter length than its predecessor). Listing the number of boxes in each row gives a partition of a non-negative integer $n$, the total number of boxes of the diagram. A Young tableau is obtained by filling in the boxes of the Young diagram with symbols taken from some alphabet, which is usually required to be a totally ordered set. Usually the alphabet consists of the first natural numbers.

Let us assume now that the set of indices is separated into two disjoint subsets: the even and the odd indices. A tableau is called semistandard or superstandard if the following conditions are satisfied:

- The entries are non decreasing along each row. 
- The rows have no repeated even entries.

- The entries are nondecreasing down each column.

- The columns have no repeated odd indices.

Some authors take the opposite convention, interchanging rows and columns.

To describe the super Grassmannian, we take indices 1, 2, 3, 4, 5 with 1, 2, 3, 4 being even and 5 being odd. Each of the generators of the super Grassmannian is associated to a pair of multi-indices, that we shall write using the letterplace notation $[30,31]$. The first pair of indices indicates the rows and the second pair indicates the columns that determine the submatrix whose minor is associated to the generator. Then we have, also in terms of the semistandard tableaux,

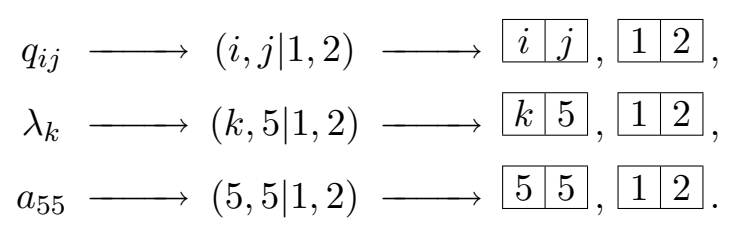

We can suppress the second tableau, which indicates the columns, since it is going to be the same in what follows. A monomial in the generators can be encoded in a tableau where each line corresponds to the indices of the coordinates, in the order in which they are written. For example, the monomials $q_{12} q_{34} \lambda_{3} a_{55}, q_{12} q_{23} \lambda_{55}$ correspond to the tableaux:

$$
\begin{array}{|l|l|}
\hline 1 & 2 \\
\hline 3 & 4 \\
\hline 3 & 5 \\
\hline 5 & 5 \\
\hline
\end{array} \quad \begin{array}{|l|l|}
\hline 1 & 2 \\
\hline 2 & 3 \\
\hline 5 & 5 \\
\hline
\end{array}
$$

The second one is semistandard, while the first one is not. As the reader can quickly note, there is in fact a relation among the coordinates whose indices appear in the first tableau: $\lambda_{3} a_{55}=0$.

The semistandard tableaux are very important since the monomials associated to them are a basis for the superring $\mathcal{O}(\mathrm{Gr})$. The next theorem provides us with a presentation of such ring and with a basis. Its proof is based on the straightening algorithm in the super setting, which we are unable to describe here, since it would take us too far from our purpose. We refer the reader to the beautiful works $[30,31]$, where the full details are discussed. 


\section{Theorem 4.1.}

1. The Grassmannian superring is given in terms of generators and relations as:

$$
\mathcal{O}(\mathrm{Gr})=\mathbb{C}\left[q_{i j}, \lambda_{j}, a_{55}\right] / \mathcal{I}_{\mathrm{P}}, \quad 1 \leq i<j \leq 5 \text { and } i=j=5
$$

where $\mathcal{I}_{\mathrm{P}}$ is the two-sided ideal generated by the super Plücker relations (4.9).

2. The Grassmannian superring is the free superring over $k$ generated by the monomials in the variables $q_{i j}, \lambda_{j}, a_{55}$ whose indeces form a semistandard tableau.

Remark 4.2. In our special case a semistandard tableaux means that the indices of the variables forming the monomial and appearing in the tableau in two consecutive lines are such that $\left(i_{k}, j_{k}\right)<\left(i_{k+1}, j_{k+1}\right)$ lexicographically, but the even pairs of type $(1,4)$ and $(2,3)$ (and more generally $(i, j),(k, l)$, with $i<k<l<j)$ are not allowed to appear: such pairs shall be disposed using the (super) Plücker relations, through the straightening algorithm [30].

The ring $\mathcal{O}(\mathrm{Gr})$ is our starting point for the quantization: we will quantize the Grassmannian together with its embedding into $\mathbf{P}(E)$ by obtaining a quantization of the ring $\mathcal{O}(\mathrm{Gr})$. We will obtain the quantized superalgebra as a sub superalgebra of the quantum supergroup $\mathrm{SL}_{q}(4 \mid 1)$. In this way, the quantized Grassmannian $\mathrm{Gr}_{q}$ will carry a natural action of the quantum supergroup $\mathrm{SL}_{q}(4 \mid 1)$, just as in the classical case.

\subsection{The conformal and Poincaré supergroups and the big cell}

We start by describing the natural action of the special linear supergroup, the conformal supergroup, on the Grassmannian supervariety.

The functor of points of the supergroup is:

$$
h_{\mathrm{SL}(4 \mid 1)}(\mathcal{A})=\left\{g=\left(\begin{array}{lllll}
c_{11} & c_{12} & c_{13} & c_{14} & \rho_{15} \\
c_{21} & c_{22} & c_{23} & c_{24} & \rho_{25} \\
c_{31} & c_{32} & c_{33} & c_{34} & \rho_{35} \\
c_{41} & c_{42} & c_{43} & c_{44} & \rho_{45} \\
\delta_{51} & \delta_{52} & \delta_{53} & \delta_{54} & d_{55}
\end{array}\right), \quad \mid \operatorname{Ber}(g)=1\right\},
$$


where $c_{i j}, d_{55} \in \mathcal{A}_{0}$ and $\rho_{i 5}, \delta_{5 i} \in \mathcal{A}_{1}$. Ber stands for the Berezinian or superdeterminant of the matrix $g$. We refer the reader to [6] Ch. 1 for its definition and main properties.

Remark 4.3. The functor $h_{\mathrm{SL}(4 \mid 1)}$ is representable, and it is represented by the algebra

$$
\mathcal{O}(\mathrm{SL}(4 \mid 1))=\mathbb{C}\left[g_{i j}, g_{55}, \gamma_{i 5}, \gamma_{5 j}\right] /(\operatorname{Ber} g-1)
$$

with $i, j=1, \ldots, 4$. Again, Latin letters are for even generators and Greek letters for odd generators. If we prefer to use the supergroup GL(4|1) we will have instead

$$
\mathcal{O}(\mathrm{GL}(4 \mid 1))=\mathbb{C}\left[g_{i j}, g_{55}, \gamma_{i 5}, \gamma_{5 j}, X, D\right] /\left(X \cdot \operatorname{det} g_{i j}-1, D \cdot g_{55}-1\right),
$$

where $X$ and $D$ are even generators.

We can describe the action of the supergroup SL(4|1) over Gr as a natural transformation of the functors,

$$
h_{\mathrm{SL}(4 \mid 1)}(\mathcal{A}) \times h_{\mathrm{Gr}}(\mathcal{A}) \longrightarrow h_{\mathrm{Gr}}(\mathcal{A}), \quad \mathcal{A} \text { local },
$$

which in this language is simply given by the multiplication of matrices (4.11) and (4.6). We leave to the reader as an exercise the fact that this definition, given for local superalgebras, can be extended to all superalgebras (see [6] Ch. 9). Also, we refer the reader to Appendix A.5 for all the definitions concerning actions and stabilizers.

As in the even case, we may take $\pi_{0}=\operatorname{span}\left\{e_{1}, e_{2}\right\}$. The stabilizer of this point is the upper parabolic sub-supergroup $P_{u}$, whose functor of points is

$$
h_{P_{u}}(\mathcal{A})=\left\{\left(\begin{array}{ccccc}
c_{11} & c_{12} & c_{13} & c_{14} & \rho_{15} \\
c_{21} & c_{22} & c_{23} & c_{24} & \rho_{25} \\
0 & 0 & c_{33} & c_{34} & \rho_{35} \\
0 & 0 & c_{43} & c_{44} & \rho_{45} \\
0 & 0 & \delta_{53} & \delta_{54} & d_{55}
\end{array}\right)\right\}
$$

Then, the Grassmannian can be identified with the quotient

$$
h_{\mathrm{Gr}}(\mathcal{A})=h_{\mathrm{SL}(4 \mid 1)}(\mathcal{A}) / h_{P_{u}}(\mathcal{A}) .
$$

The description of homogeneous spaces for super Lie groups is done in detail in [1]. 
These algebras are commutative Hopf superalgebras. The comultiplication is given as usual by matrix multiplication, (see for example [20,21], also for the counit and antipode), by organizing the generators in matrix form:

$$
\Delta\left(\begin{array}{ll}
g_{i j} & \gamma_{i 5} \\
\gamma_{5 j} & g_{55}
\end{array}\right)=\left(\begin{array}{ll}
g_{i k} & \gamma_{i 5} \\
\gamma_{5 k} & g_{55}
\end{array}\right) \otimes\left(\begin{array}{ll}
g_{k j} & \gamma_{k 5} \\
\gamma_{5 j} & g_{55}
\end{array}\right)
$$

The superalgebra $\mathcal{O}(\mathrm{Gr})$ is a sub superalgebra (not a Hopf sub superalgebra) of $\mathcal{O}(\mathrm{SL}(4 \mid 1))$. It is in fact the superalgebra generated by the corresponding minors, and the Plücker relations are all the relations satisfied by these minors in $\mathcal{O}(\mathrm{SL}(4 \mid 1))$.

As in the nonsuper case, the super Grassmannian admits an open cover in terms of affine superspaces. In terms of the functor of points we say that Gr admits a cover by "open affine subfunctors". This is explained in detail in the Appendix A.4, and it generalizes the open cover of the nonsuper case given in Section 4.1. As we have detailed in the previous section, we shall concentrate our attention just on local algebras. We will describe $h_{U_{12}}$, the functor of points of the big cell $U_{12}$. First of all, we write an element of $h_{\mathrm{SL}(4 \mid 1)}(\mathcal{A})$ in blocks as (see $\left.(4.11)\right)$

$$
\left(\begin{array}{ccc}
C_{1} & C_{2} & \rho_{1} \\
C_{3} & C_{4} & \rho_{2} \\
\delta_{1} & \delta_{2} & d_{55}
\end{array}\right) .
$$

Assuming that $\operatorname{det} C_{1}$ is invertible, we can bring this matrix, with a transformation of $h_{P_{u}}(\mathcal{A})$, to the form

$$
\left(\begin{array}{ccc}
\mathbb{1}_{2} & 0 & 0 \\
A & \mathbb{1}_{2} & 0 \\
\alpha & 0 & 1
\end{array}\right) .
$$

The assumption that $\operatorname{det} C_{1}$ invertible means that the matrix is in the open set $U_{12}=\operatorname{Gr} \cap V_{12}$, where $V_{12}$ is the affine open set defined by taking in $\mathbf{P}(E)$ the coordinate $q_{12} \neq 0$.

So if the column vectors of

$$
\left(\begin{array}{l}
C_{1} \\
C_{3} \\
\delta_{1}
\end{array}\right)
$$


with $\operatorname{det} C_{1}$ invertible represent a $2 \mid 0$-module in the big cell, the same module can be represented by a matrix of the form

$$
\left(\begin{array}{c}
\mathbb{1}_{2} \\
A \\
\alpha
\end{array}\right), \quad A=\left(\begin{array}{ll}
a_{11} & a_{12} \\
a_{21} & a_{22}
\end{array}\right), \quad \alpha=\left(\alpha_{1}, \alpha_{2}\right),
$$

with the entries of $A$ in $\mathcal{A}_{0}$ and the entries of $\alpha$ in $\mathcal{A}_{1}$. The big cell $U_{12}$ of $\mathrm{Gr}$ is then the affine superspace associated with the superalgebra

$$
\mathcal{O}\left(U_{12}\right)=\mathbb{C}\left[a_{i j}, \alpha_{j}\right] \approx \mathbb{C}^{4 \mid 2} .
$$

We are now interested in the subsupergroup of $h_{\mathrm{SL}(4 \mid 1)}(\mathcal{A})$ that preserves the big cell $U_{12}$. This is the stabilizer functor $\operatorname{Stab}_{U_{12}}$. According to Definition A.9 we have

$$
\begin{aligned}
\operatorname{Stab}_{U_{12}}(\mathcal{A})= & \left\{g \in h_{\mathrm{SL}(4 \mid 1)}(\mathcal{A}) \mid g \cdot h_{U_{12}}\left(\mathcal{A}^{\prime}\right)\right. \\
& \left.\subset h_{U_{12}}\left(\mathcal{A}^{\prime}\right) \text { for all } \mathcal{A} \text {-algebras } \mathcal{A}^{\prime}\right\} .
\end{aligned}
$$

As we remarked after Definition A.9, Stab $_{U_{12}}$ is not in general representable, and it does not correspond to the functor of points of a supervariety. Nevertheless, there exists a subsupergroup $\mathrm{St}_{U_{12}}$ of $\mathrm{SL}(4 \mid 1)$, whose functor of points is the largest subgroup functor of $\mathrm{Stab}_{U_{12}}$.

In our case, $\mathrm{St}_{U_{12}}$ is the lower parabolic sub supergroup $P_{1}$, whose functor of points is given in suitable coordinates by matrices of the type

$$
h_{P_{1}}(\mathcal{A})=\left\{\left(\begin{array}{ccc}
x & 0 & 0 \\
t x & y & y \eta \\
d \tau & d \xi & d
\end{array}\right)\right\}
$$

where $x$ and $y$ are even, invertible $2 \times 2$ matrices, $t$ is an even, arbitrary $2 \times 2$ matrix, $\eta$ a $2 \times 1$ odd matrix and $\tau$ and $\xi$ are $1 \times 2$ odd matrices. $d$ is an invertible even element given by the superdeterminant equal 1 condition.

Let us see this. We clearly have from the definitions

$$
h_{P_{1}}(\mathcal{A}) \subset h_{\mathrm{St}_{U_{12}}}(\mathcal{A}) .
$$

Since this embedding is functorial, we have an embedding $P_{1} \subset \mathrm{St}_{U_{12}}$ of the algebraic supergroups. Since any supergroup over $\mathbb{C}$ is also a supermanifold (see [29]) we have that this is also a supermanifold embedding. 
Let us look at the superdimension of the stabilizer $\mathrm{St}_{U_{12}}$ and $P_{1}$. The superdimension is well defined since these are supermanifolds. To compute them one can look at the tangent spaces. We then have

$$
\operatorname{dim} \mathrm{St}_{U_{12}} \leq \operatorname{dim} \mathrm{SL}(4 \mid 1)-\operatorname{dim} U_{12}=4^{2}+1|2 \cdot 4-4| 2=13 \mid 6,
$$

but

$$
\operatorname{dim} P_{1}=2^{2}+2^{2}+2^{2}+1|2+2+2=13| 6,
$$

hence $\operatorname{dim} \mathrm{St}_{U_{12}}=\operatorname{dim} P_{1}$. Now, the equality $\mathrm{St}_{U_{12}}=P_{1}$ follows from the following theorem.

Theorem 4.2. Let $M$ and $N$ be two supermanifolds with the same dimension and such that $|M|=|N|$. If we have an embedding $M \subset N$ then $M \cong N$.

Proof. See in [6] Ch. 5.

The action of the stabilizer supergroup $P_{1}$ on the big cell $U_{12}$ is as follows,

$$
\begin{gathered}
h_{P_{1}}(\mathcal{A}) \times h_{U_{12}}(\mathcal{A}) \\
\left.\left(\begin{array}{ccc}
x & 0 & 0 \\
t x & y & y \eta \\
d \tau & d \xi & d
\end{array}\right),\left(\begin{array}{c}
\mathbb{1}_{2} \\
A \\
\alpha
\end{array}\right)\right) \longrightarrow h_{U_{12}}(\mathcal{A}), \\
\longrightarrow\left(\begin{array}{c}
\mathbb{1}_{2} \\
A^{\prime} \\
\alpha^{\prime}
\end{array}\right),
\end{gathered}
$$

where, using a transformation of $h_{P_{u}}(\mathcal{A})$ to revert the resulting matrix to the standard form (4.14), we have

$$
\left(\begin{array}{c}
\mathbb{1}_{2} \\
A^{\prime} \\
\alpha^{\prime}
\end{array}\right)=\left(\begin{array}{c}
\mathbb{1}_{2} \\
y(A+\eta \alpha) x^{-1}+t \\
d(\alpha+\tau+\xi A) x^{-1}
\end{array}\right) .
$$

The subgroup with $\xi=0$ is the super Poincaré group times dilations (compare with equation (14) in [1]). In that case

$$
d=\operatorname{det} x \operatorname{det} y .
$$

\section{Quantum super Grassmannian}

Before giving the definition of quantum super Grassmannian, we need some preliminaries on quantum supergroups in general. 


\subsection{Quantum supergroups}

In this section, we follow Manin [19].

Definition 5.1. The quantum matrix superalgebra $\mathrm{M}_{q}(m \mid n)$ is defined as

$$
\mathrm{M}_{q}(m \mid n)=_{\text {def }} \mathbb{C}_{q}\left\langle x_{i j}, \xi_{k l}\right\rangle / \mathcal{I}_{M},
$$

where $\mathbb{C}_{q}\left\langle x_{i j}, \xi_{k l}\right\rangle$ denotes the free superalgebra over $\mathbb{C}_{q}=\mathbb{C}\left[q, q^{-1}\right]$ generated by the even variables

$$
x_{i j}, \quad \text { for } 1 \leq i, j \leq m \text { or } m+1 \leq i, j \leq m+n .
$$

and by the odd variables

$$
\begin{aligned}
\xi_{k l} \quad & \text { for } 1 \leq k \leq m, \quad m+1 \leq l \leq m+n, \\
& \text { or } m+1 \leq k \leq m+n, \quad 1 \leq l \leq m,
\end{aligned}
$$

satisfying the relations $\xi_{k l}^{2}=0 . \mathcal{I}_{M}$ is an ideal generated by relations that we will describe shortly.

To simplify the notation it is convenient sometimes to have a common notation for even and odd variables

$$
a_{i j}= \begin{cases}x_{i j} & 1 \leq i, j \leq m, \quad \text { or } \quad m+1 \leq i, j \leq m+n, \\ \xi_{i j} \quad 1 \leq i \leq m, \quad m+1 \leq j \leq m+n, & \text { or } \\ & m+1 \leq i \leq m+n, \quad 1 \leq j \leq m .\end{cases}
$$

The ideal $\mathcal{I}_{M}$ is generated by the relations [19]:

$$
\begin{array}{ll}
a_{i j} a_{i l}=(-1)^{\pi\left(a_{i j}\right) \pi\left(a_{i l}\right)} q^{(-1)^{p(i)+1}} a_{i l} a_{i j}, & \text { for } j<l, \\
a_{i j} a_{k j}=(-1)^{\pi\left(a_{i j}\right) \pi\left(a_{k j}\right)} q^{(-1)^{p(j)+1}} a_{k j} a_{i j}, & \text { for } i<k,
\end{array}
$$




$$
\begin{array}{ll}
a_{i j} a_{k l}=(-1)^{\pi\left(a_{i j}\right) \pi\left(a_{k l}\right)} a_{k l} a_{i j}, & \text { for } i<k, j>l, \\
a_{i j} a_{k l}-(-1)^{\pi\left(a_{i j}\right) \pi\left(a_{k l}\right)} a_{k l} a_{i j} & \text { or } i>k, j<l, \\
=(-1)^{\pi\left(a_{i j}\right) \pi\left(a_{k j}\right)}\left(q^{-1}-q\right) a_{k j} a_{i l}, & \text { for } i<k, j<l,
\end{array}
$$

where $\pi\left(a_{i j}\right)=p(i)+p(j) \bmod 2$ denotes the parity of $a_{i j}$ (with $p(i)=0$ if $1 \leq i \leq m$ and $p(i)=1$ otherwise).

Remark 5.1. In our definition we take $q$ to be an indeterminate. However this definition makes sense also for any value of $q \in \mathbb{C}^{\times}$(usually one asks away from roots of one). In any case, whenever we make use of the fact that $q$ needs to be an indeterminate we will say it.

$\mathrm{M}_{q}(m \mid n)$ is a super bialgebra with the usual comultiplication and counit:

$$
\Delta\left(a_{i j}\right)=\sum a_{i k} \otimes a_{k j}, \quad \mathcal{E}\left(a_{i j}\right)=\delta_{i j} .
$$

Note that the comultiplication, which encodes the matrix product law (and then the group law), is not deformed. In fact $\mathrm{M}(m \mid n)$ is a bialgebra with the commutative product $\left(q=1\right.$ in $\left.\mathcal{I}_{M}\right)$ and the same comultiplication and counit. For later use, we will display here the commutative diagram for the coassociativity axiom satisfied by the comultiplication,

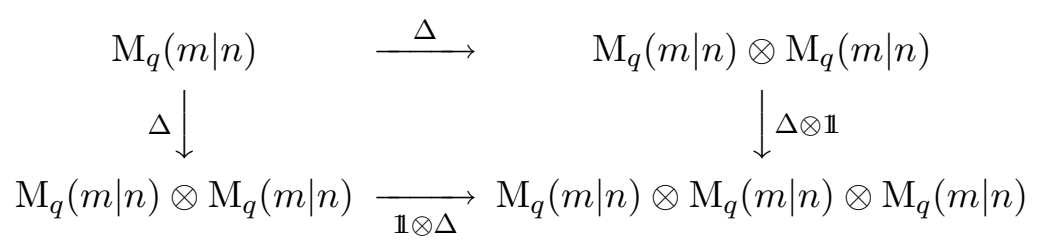

We are ready to define the general linear supergroup. Let

$$
\begin{aligned}
& D_{1}={ }_{\operatorname{def}} \sum_{\sigma \in S_{m}}(-q)^{-l(\sigma)} a_{1 \sigma(1)} \ldots a_{m \sigma(m)} \\
& D_{2}={ }_{\operatorname{def}} \sum_{\sigma \in S_{n}}(-q)^{l(\sigma)} a_{m+1, m+\sigma(1)} \ldots a_{m+n, m+\sigma(n)}
\end{aligned}
$$

be the quantum determinants of the diagonal blocks. 
Definition 5.2. The quantum general linear supergroup $\mathrm{GL}_{q}(m \mid n)$ is defined as

$$
\mathrm{GL}_{q}(m \mid n)={ }_{\operatorname{def}} \mathrm{M}_{q}(m \mid n)\left[D_{1}^{-1}, D_{2}^{-1}\right],
$$

where $D_{1}^{-1}$ and $D_{2}^{-1}$ are even indeterminates such that

$$
\begin{gathered}
D_{1} D_{1}^{-1}=1=D_{1}^{-1} D_{1}, \\
D_{2} D_{2}^{-1}=1=D_{2}^{-1} D_{2} .
\end{gathered}
$$

The quantum general linear supergroup $\mathrm{GL}_{q}(m \mid n)$ is defined as

$$
\mathrm{SL}_{q}(m \mid n)={ }_{\text {def }} \mathrm{M}_{q}(m \mid n) /\left\langle\operatorname{Ber}_{q}-1\right\rangle,
$$

where $\operatorname{Ber}_{q}$ is the quantum Berezinian (for its definition and properties refer to $[21])$.

$\mathrm{GL}_{q}(m \mid n)$ and $\mathrm{SL}_{q}(m \mid n)$ are Hopf superalgebras. The comultiplication and the counit are the same as in $\mathrm{M}_{q}(m \mid n)$. One must give also the comultiplication on $D_{1}^{-1}$ and $D_{2}^{-1}$, and the antipode $S$. This has been done in detail in $[20,21,41]$.

The roles of $\mathrm{GL}_{q}(m \mid n)$ and $\mathrm{SL}_{q}(m \mid n)$ can be interchanged in what follows, as it happens for the super nonquantum setting. We then make the choice to use $\mathrm{SL}_{q}(m \mid n)$.

\subsection{Presentation of the quantum super Grassmannian $\mathrm{Gr}_{q}$}

Let the notation be as above. We are going to define the quantum super Grassmannian $\mathrm{Gr}_{q}$ in terms of generators and relations. Then we will prove that it is a deformation of the algebra $\mathcal{O}(\mathrm{Gr})$ given in (4.10). We are now ready for the central definition of our paper, namely the quantum deformation of the super Grassmannian.

Definition 5.3. The quantum super Grassmannian of $2 \mid 0$ planes in $4 \mid 1$ dimensional superspace is the noncommutative superalgebra $\mathrm{Gr}_{q}$ generated by the following quantum super minors in $\mathrm{SL}_{q}(4 \mid 1)$ :

$$
\begin{aligned}
& D_{i j}=a_{i 1} a_{j 2}-q^{-1} a_{i 2} a_{j 1}, \quad 1 \leq i<j \leq 4, \\
& D_{i 5}=a_{i 1} a_{52}-q^{-1} a_{i 2} a_{51}, \quad 1 \leq i \leq 4 \\
& D_{55}=a_{51} a_{52} \text {. }
\end{aligned}
$$


For clarity we write explicitly all the generators:

$$
\begin{aligned}
& D_{12}, \quad D_{13}, \quad D_{14}, \quad D_{23}, \quad D_{24}, \quad D_{34}, \quad D_{55}, \quad \text { (even), } \\
& D_{15}, \quad D_{25}, \quad D_{35}, \quad D_{45} \quad \text { (odd). }
\end{aligned}
$$

Note that $D_{55}$ is an even nilpotent element.

The parity is easily given by the rule:

$$
\left|D_{i j}\right|=(-1)^{|i|+|j|}, \quad|i|=0 \text { for } i=1, \ldots 4, \text { and }|i|=1 \text { for } i=5 \text {. }
$$

We will see that this subalgebra of $\mathrm{SL}_{q}(4 \mid 1)$ is generated, as a vector space, by monomials in the above determinants. This fact is not obvious at all, since the commutation relations of the minors could introduce terms that are not included in (5.3). For $q=1$, we recover the classical Grassmannian algebra $\mathcal{O}(\mathrm{Gr})$ described in detail in Section 4.2.

More precisely, we will find a presentation of $\mathrm{Gr}_{q}$ in terms of generators $X_{i j}$ (identified with the $D_{i j}$ in (5.3)) and relations. In order to do so, we first work out the commutation relations of the minors. Then, as in the classical setting, there will be additional relations among the generators: the quantum super Plücker relations.

Let us start with the commutation relations. In [7] such commutation relations are given for the even minors $D_{i j}$, with $1 \leq i<j \leq 4$. As one can readily check, they hold also when just one of the indices is 5 . The reason is that the commutation of one even and one odd variable in the matrix bialgebra generated by the $a_{i j}$ 's is the same as the commutation of two even variables, and the expression of $D_{k l}$ in terms of $a_{i j}$ is formally the same.

The commutation relations are as follows:

- If $i, j, k, l$ are not all distinct we have:

$$
D_{i j} D_{k l}=q^{-1} D_{k l} D_{i j}, \quad(i, j)<(k, l), \quad 1 \leq i<j<k<l \leq 5
$$

where " $<$ " refers to the lexicographic ordering.

- If $i, j, k, l$ are all distinct we have:

$$
\begin{array}{ll}
D_{i j} D_{k l}=q^{-2} D_{k l} D_{i j}, & 1 \leq i<j<k<l \leq 5, \\
D_{i j} D_{k l}=q^{-2} D_{k l} D_{i j}-\left(q^{-1}-q\right) D_{i k} D_{j l}, & 1 \leq i<k<j<l \leq 5, \\
D_{i j} D_{k l}=D_{k l} D_{i j}, & 1 \leq i<k<l<j \leq 5 .
\end{array}
$$


So the only quantum commutation relations that have to be computed are for

$$
D_{i j} D_{55}, \quad D_{i 5} D_{j 5}, \quad D_{i 5} D_{55} .
$$

After some computations one gets (for $1 \leq i<j \leq 4)$ :

$$
\begin{aligned}
& D_{i j} D_{55}=q^{-2} D_{55} D_{i j}, \\
& D_{i 5} D_{j 5}=-q^{-1} D_{j 5} D_{i 5}-\left(q^{-1}-q\right) D_{i j} D_{55}=-q D_{j 5} D_{i 5}, \\
& D_{i 5} D_{55}=D_{55} D_{i 5}=0 .
\end{aligned}
$$

This concludes the discussion of the commutation relations. We are ready to tackle the calculation of the quantum super Plücker relations.

Again using the result for the nonsuper setting ([7]) we have

$$
\begin{aligned}
& D_{12} D_{34}-q^{-1} D_{13} D_{24}+q^{-2} D_{14} D_{23}=0, \\
& D_{i j} D_{k 5}-q^{-1} D_{i k} D_{j 5}+q^{-2} D_{i 5} D_{j k}=0, \quad 1 \leq i<j<k \leq 4,
\end{aligned}
$$

which yield a total of 5 relations. To these we must add the relations, which can be computed directly:

$$
D_{i 5} D_{j 5}=q D_{i j} D_{55}, \quad 1 \leq i<j \leq 4 .
$$

The relations $(5.7,5.8)$ are the quantum super Plücker relations. If one specifies $q=1$, the superalgebra becomes commutative and the quantum super Plücker relations become the standard ones (4.9).

Now we want to show that the commutation relations together with the quantum super Plücker relations are all the relations among the determinants. We do this in the following proposition.

\section{Proposition 5.1.}

(1) The quantum Grassmannian superring is given in terms of generators and relations as:

$$
\mathrm{Gr}_{q}=\mathbb{C}_{q}\left\langle X_{i j}\right\rangle / I_{\mathrm{Gr}}, \quad 1 \leq i<j \leq 5 \quad \text { and } \quad i=j=5,
$$

where $I_{\mathrm{Gr}}$ is the two-sided ideal generated by the commutations relations $(5.4,5.5,5.6)$ and the quantum super Plücker relations $(5.7,5.8)$ where $D_{i j}$ is substituted by the indeterminates $X_{i j}$. Moreover $\mathrm{Gr}_{q} /(q-$ $1) \cong \mathcal{O}(\mathrm{Gr})$ (see Section 4.2). 
(2) The quantum Grassmannian ring is the free ring over $\mathbb{C}_{q}$ generated by the monomials in the quantum determinants:

$$
D_{i_{1} j_{1}} \cdots D_{i_{r} j_{r}},
$$

where $\left(i_{1}, j_{1}\right), \ldots,\left(i_{r}, j_{r}\right)$ form a semistandard tableau (see (4.1)).

Proof. Though the proof is based on the classical result and it is the same as [34] we briefly sketch it, since its importance in our construction.

The generic monomials in the quantum determinants $D_{i j}$ generate the ring $\mathrm{Gr}_{q}$ as $\mathbb{C}_{q}$-module. Using the commutation relations we can certainly write any monomial as a lexicographically ordered monomial, then using the quantum Plücker relations and the straightening algorithm [40], we can rewrite any lexicographically ordered monomial as a linear combination of standard monomials.

Note that there are two obstacles to apply the straightening algorithm to the quantum setting, but they are both easily overcome. The first is the presence of the coefficients $q$ in the Plücker relations. This is not a problem, since $q$ is invertible. The second is the noncommutativity: when we are commuting two quantum determinants one may argue that the pairs of the forbidden kind, that is $(i, j)$ and $(k, l)$ with $i<k<l<j$ may arise. However, a closer look to the commutation relations shows that this is never the case.

So both (1) and (2) will be done if we can show that the standard monomials in the $D_{i j}$ 's are linearly independent (i.e., there are no other relations among them apart the $I_{\mathrm{Gr}}$ ones). Assume that there is a relation $R$ among such monomials. Clearly $R=0 \bmod (q-1)$ since there are no relations among the standard classical monomials, hence $R=(q-1) R^{\prime}$. Such relation evidently holds also in the bigger superalgebra $\mathrm{SL}_{q}(m \mid n)$, which is known to be torsion free. Hence the quantum determinants satisfy $R^{\prime}$ and repeating this same argument enough times we obtain a nontrivial relation among the classical monomials, hence the relation $R$ we start with cannot exist.

\section{3 $\quad \mathrm{Gr}_{q}$ as a homogeneous quantum space}

We want to prove that the quantum super Grassmannian that we have constructed admits a coaction of a quantum group on it, namely the quantum group $\mathrm{SL}_{q}(4 \mid 1)$, as it happens in the classical setting. 
Proposition 5.2. $\mathrm{Gr}_{q}$ is a quantum homogeneous superspace for the quantum supergroup $\mathrm{SL}_{q}(4 \mid 1)$, i.e., there is a coaction on $\mathrm{Gr}_{q}$ given via the restriction of the comultiplication on $\mathrm{SL}_{q}(4 \mid 1)$ (5.1):

$$
\left.\Delta\right|_{\mathrm{Gr}_{q}}: \mathrm{Gr}_{q} \longrightarrow \mathrm{SL}_{q}(4 \mid 1) \otimes \mathrm{Gr}_{q}
$$

Proof. We just have to check that the restriction is well defined. Then, the coaction property

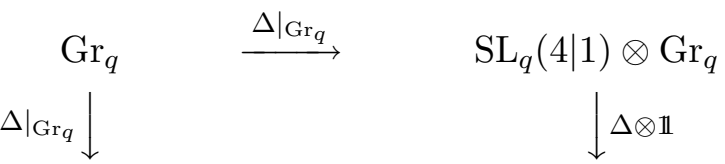

$$
\begin{aligned}
& \mathrm{SL}_{q}(4 \mid 1) \otimes_{\mathrm{Gr}_{q}} \underset{\left.\mathbb{1} \otimes \Delta\right|_{\mathrm{Gr}_{q}}}{\longrightarrow} \mathrm{SL}_{q}(4 \mid 1) \otimes \mathrm{SL}_{q}(4 \mid 1) \otimes \mathrm{Gr}_{q}
\end{aligned}
$$

is guaranteed by the coassociativity (5.2).

So we need to verify that

$$
\Delta\left(D_{i j}\right), \quad \Delta\left(D_{i 5}\right), \quad \Delta\left(D_{55}\right) \quad \in \quad \mathrm{SL}_{q}(4 \mid 1) \otimes \mathrm{Gr}_{q} .
$$

Let us denote the generic $2 \times 2$ quantum minor as

$$
D_{i j}^{k l}=a_{i k} a_{j l}-q^{-1} a_{i l} a_{j k},
$$

so in the previous notation $D_{i j}=D_{i j}^{12}$. In the purely even setting, we can use the formula (see [7])

$$
\Delta\left(D_{i j}^{12}\right)=\sum_{1 \leq k<l \leq 4} D_{i j}^{k l} \otimes D_{k l}^{12} .
$$

In the super case, we can extend the sum to $l=5$. Also for the minors $D_{i 5}$ (the only odd ones) the calculation is the same. This proves immediately that

$$
\Delta\left(D_{i j}\right), \Delta\left(D_{i 5}\right) \in \mathrm{SL}_{q}(4 \mid 1) \otimes \mathrm{Gr}_{q}, \text { for } 1 \leq i<j \leq 4 .
$$

For $\Delta\left(D_{55}\right)$ it is a straightforward (long) check.

\section{Quantum deformation of the big cell inside the super Grassmannian}

We want now to define the analogue, in the quantum setting, of the superalgebra representing the big cell of the Grassmannian supermanifold. At the classical level we obtained it in (4.15). 
The superalgebra $\mathcal{O}\left(U_{12}\right) \approx \mathbb{C}^{4 \mid 2}$ is the superalgebra corresponding to the chiral Minkowski superspace (see Section 2). In Section 4.4, we wrote the action of the lower parabolic supergroup $P_{1}$ (which includes the super Poincaré group times dilations) using the functor of points (4.17). We want now to translate it into the coaction language to make the generalization to the quantum setting. The first step is to understand the Hopf superalgebra of the lower parabolic supergroup $P_{1}$ as the quotient, by a suitable ideal, of the algebra representing $\mathrm{SL}(4 \mid 1)$

$$
\mathcal{O}(\mathrm{SL}(4 \mid 1))=\mathbb{C}\left[g_{i j}, g_{55}, \gamma_{i 5}, \gamma_{5 j}\right] /\left(\mathrm{Ber}_{q}-1\right)
$$

(see equation (4.12)). The generators can be written in matrix form

$$
\left(\begin{array}{ll}
g_{i j} & \gamma_{i 5} \\
\gamma_{5 j} & g_{55}
\end{array}\right)
$$

so to read the comultiplication as the matrix product.

Proposition 6.1. Let $\mathcal{O}\left(P_{1}\right)$ be the superalgebra:

$$
\mathcal{O}\left(P_{1}\right):=\mathcal{O}(\mathrm{SL}(4 \mid 1)) / \mathcal{I}
$$

where $\mathcal{I}$ is the (two-sided) ideal generated by

$$
g_{1 j}, g_{2 j}, \quad \text { for } j=3,4 \text { and } \gamma_{15}, \gamma_{25} .
$$

This is the Hopf superalgebra of the lower parabolic subgroup, with comultiplication naturally inherited by $\mathcal{O}(\mathrm{SL}(4 \mid 1))$.

Proof. One can readily check that $\mathcal{I}$ is a Hopf ideal, in other words

$$
\Delta(\mathcal{I})=\mathcal{I} \otimes \mathcal{O}(\mathrm{SL}(4 \mid 1))+\mathcal{O}(\mathrm{SL}(4 \mid 1)) \otimes \mathcal{I} .
$$

Hence the Hopf superalgebra structure goes to the quotient. The fact that $\mathcal{O}\left(P_{1}\right)$ represents the lower parabolic supergroup $P_{1}$ is also clear.

In matrix form, for $\mathcal{A}$ local, we have

$$
h_{P_{1}}(\mathcal{A})=\left\{\left(\begin{array}{ccccc}
g_{11} & g_{12} & 0 & 0 & 0 \\
g_{21} & g_{22} & 0 & 0 & 0 \\
g_{31} & g_{32} & g_{33} & g_{34} & \gamma_{35} \\
g_{41} & g_{42} & g_{43} & g_{44} & \gamma_{45} \\
\gamma_{51} & \gamma_{52} & \gamma_{53} & \gamma_{54} & g_{55}
\end{array}\right)\right\} \subset h_{\mathrm{SL}(m \mid n)}(\mathcal{A}) .
$$

The superalgebra representing the big cell is in fact a sub superalgebra (not a Hopf subalgebra) of $\mathcal{O}\left(P_{1}\right)$. It is more convenient to make a change of 
variables for the generators, so

$$
\left(\begin{array}{ccccc}
g_{11} & g_{12} & 0 & 0 & 0 \\
g_{21} & g_{22} & 0 & 0 & 0 \\
g_{31} & g_{32} & g_{33} & g_{34} & \gamma_{35} \\
g_{41} & g_{42} & g_{43} & g_{44} & \gamma_{45} \\
\gamma_{51} & \gamma_{52} & \gamma_{53} & \gamma_{54} & g_{55}
\end{array}\right)=\left(\begin{array}{ccc}
x & 0 & 0 \\
t x & y & y \eta \\
\tilde{\tau} x & d \xi & d
\end{array}\right)
$$

The notation used here is slightly different to the notation used in (4.16). We can define

$$
d \tau=\tilde{\tau} x
$$

but we will see that having $\tilde{\tau}$ is essential to describe the bigcell. With this change of variable we have:

$$
h_{P_{1}}(\mathcal{A})=\left(\begin{array}{ccc}
x & 0 & 0 \\
t x & y & y \eta \\
d \tau & d \xi & d
\end{array}\right)
$$

Proposition 6.2. The Hopf superalgebra $\mathcal{O}\left(P_{1}\right)$ is generated by the two alternative set of variables:

- $x, y, t, \tilde{\tau}, \xi, \eta$ and $d$,

- $x, y, t, \tau, \xi, \eta$ and $d$.

The sub superalgebra of $\mathcal{O}\left(P_{1}\right)$ generated by $(t, \tilde{\tau})$ coincides with the big cell superring $\mathcal{O}\left(U_{12}\right)$ as defined in (4.15).

Moreover, there is a well defined coaction of $\mathcal{O}\left(P_{1}\right)$ on $\mathcal{O}\left(U_{12}\right)$ induced by the comultiplication (6.3),

$$
\tilde{\Delta}: \mathcal{O}\left(U_{12}\right) \longrightarrow \mathcal{O}\left(P_{1}\right) \otimes \mathcal{O}\left(U_{12}\right)
$$

which explicitly takes the form:

$$
\begin{aligned}
& \tilde{\Delta} t_{i j}=t_{i j} \otimes 1+y_{i a} S(x)_{b j} \otimes t_{a b}+y_{i} \eta_{a} S(x)_{b j} \otimes \tilde{\tau}_{j b}, \\
& \tilde{\Delta} \tilde{\tau}_{j}=(d \otimes 1)\left(\tau_{a} \otimes 1+\xi_{b} \otimes t_{b a}+1 \otimes \tilde{\tau}_{a}\right)\left(S(x)_{a j} \otimes 1\right) .
\end{aligned}
$$

(The reader should notice right away that this is the dual to the expression $(4.17))$.

Proof. First of all it is clear that the two given sets generate the superring $\mathcal{O}\left(P_{1}\right)$. The comultiplication of the new variables can be computed in terms 
of the comultiplication of the old variables

$$
\Delta\left(\begin{array}{ccc}
x & 0 & 0 \\
t x & y & y \eta \\
\tilde{\tau} x & d \xi & d
\end{array}\right)=\left(\begin{array}{ccc}
x & 0 & 0 \\
t x & y & y \eta \\
\tilde{\tau} x & d \xi & d
\end{array}\right) \otimes\left(\begin{array}{ccc}
x & 0 & 0 \\
t x & y & y \eta \\
\tilde{\tau} x & d \xi & d
\end{array}\right)
$$

From (6.3) we have that

$$
\begin{aligned}
\Delta x & =x \otimes x, \\
\Delta(t x) & =t x \otimes x+y \otimes t x+y \eta \otimes \tau x, \\
\Delta(\tilde{\tau} x) & =\tau x \otimes x+d \xi \otimes t x+d \otimes \tau x .
\end{aligned}
$$

It is convenient to write this in component form. From (6.3) we have that

$$
\begin{aligned}
\Delta x_{i j} & =x_{i k} \otimes x_{k j}, \\
\Delta\left(t_{i j} x_{j l}\right) & =\left(\Delta t_{i j}\right)\left(\Delta x_{j l}\right)=t_{i a} x_{a b} \otimes x_{b l}+y_{i a} \otimes t_{a b} x_{b l}+y_{i a} \eta_{a} \otimes \tilde{\tau}_{j b} x_{b} \\
& =\left(t_{i j} \otimes 1+y_{i a} S(x)_{b j} \otimes t_{a b}+y_{i} \eta_{a} S(x)_{b j} \otimes \tilde{\tau}_{j b}\right)\left(x_{j p} \otimes x_{p l}\right), \\
\Delta\left(\tilde{\tau}_{j} x_{j l}\right) & =\left(\Delta \tilde{\tau}_{j}\right) \otimes\left(\Delta x_{j l}\right)=\tilde{\tau}_{j} x_{j k} \otimes x_{k l}+d \xi_{j} \otimes t_{j k} x_{k l}+d \otimes \tilde{\tau}_{j} x_{j l} \\
& =\left(\tilde{\tau}_{j} \otimes 1+d \xi_{b} S(x)_{a j} \otimes t_{b a}+d S(x)_{a j} \otimes \tilde{\tau}_{a}\right)\left(x_{j k} \otimes x_{k l}\right),
\end{aligned}
$$

where $S$ is the antipode,

$$
S(x)=x^{-1}=\frac{1}{\operatorname{det}(x)}\left(\begin{array}{cc}
x_{22} & -x_{12} \\
-x_{21} & x_{11}
\end{array}\right) .
$$

From these equations we can read the coaction of the group on the big cell,

$$
\begin{aligned}
\tilde{\Delta} t_{i j} & =t_{i j} \otimes 1+y_{i a} S(x)_{b j} \otimes t_{a b}+y_{i} \eta_{a} S(x)_{b j} \otimes \tilde{\tau}_{j b}, \\
\tilde{\Delta} \tilde{\tau}_{j} & =\tilde{\tau}_{j} \otimes 1+d \xi_{b} S(x)_{a j} \otimes t_{b a}+d S(x)_{a j} \otimes \tilde{\tau}_{a} .
\end{aligned}
$$

It is straightforward now to compare (6.5) with (4.17) to realize that the action and the coaction are dual to each other. It is enough to use (6.2) but only in the first factor of the tensor product:

$$
\begin{aligned}
\tilde{\Delta} \tilde{\tau}_{j} & =d \tau_{a} S(x)_{a j} \otimes 1+d \xi_{b} S(x)_{a j} \otimes t_{b a}+d S(x)_{a j} \otimes \tilde{\tau}_{a} \\
& =(d \otimes 1)\left(\tau_{a} \otimes 1+\xi_{b} \otimes t_{b a}+1 \otimes \tilde{\tau}_{a}\right)\left(S(x)_{a j} \otimes 1\right),
\end{aligned}
$$

and we obtain the coaction in the desired form.

We now turn to the quantum setting. We shall repeat all the classical arguments, exerting however extreme care, since in all of our calculations, 
the Manin commutation relations (see Definition 5.1) now play a key role. In order to keep our notation minimal we use the same letters as in the classical case to denote the generators of the quantum big cell and the quantum supergroups.

Proposition 6.3. Let $\mathcal{O}\left(P_{l, q}\right)$ be the superalgebra:

$$
\mathcal{O}\left(P_{l, q}\right):=\mathcal{O}\left(\mathrm{SL}_{q}(4 \mid 1)\right) / \mathcal{I}_{q}
$$

where $\mathcal{I}_{q}$ is the (two-sided) ideal in $\mathcal{O}\left(\mathrm{SL}_{q}(4 \mid 1)\right)$ generated by

$$
g_{1 j}, g_{2 j}, \quad \text { for } j=3,4 \text { and } \gamma_{15}, \gamma_{25}
$$

This is the Hopf superalgebra of the quantum lower parabolic subgroup, with comultiplication the one naturally inherited from $\mathcal{O}\left(\mathrm{SL}_{q}(4 \mid 1)\right)$.

Proof. Note that the comultiplication is the same than in the classical case, so $\mathcal{I}_{q}$ is a Hopf ideal (see Proposition 6.1) and the Hopf superalgebra structure goes to the quotient.

Remark 6.1. The quantum lower parabolic supergroup is generated by the images in the quotient of the generators $g_{i j}$ and $\gamma_{i j}$ that are not listed in (6.6). In the quantum case, this is a nontrivial fact, because in the commutation relations among the generators of the ideal may appear generators other than the ones in (6.6), giving then a "bigger" ideal than in the classical case. One can check that this does not happen here (see for example [32]).

As in the classical case, it is convenient to change coordinates

$$
\left(\begin{array}{ccccc}
g_{11} & g_{12} & 0 & 0 & 0 \\
g_{21} & g_{22} & 0 & 0 & 0 \\
g_{31} & g_{32} & g_{33} & g_{34} & \gamma_{35} \\
g_{41} & g_{42} & g_{43} & g_{44} & \gamma_{45} \\
\gamma_{51} & \gamma_{52} & \gamma_{53} & \gamma_{54} & g_{55}
\end{array}\right)=\left(\begin{array}{ccc}
x & 0 & 0 \\
t x & y & y \eta \\
\tilde{\tau} x & d \xi & d
\end{array}\right)
$$

Note that in $\mathcal{O}\left(P_{l, q}\right)$ the elements $D_{12}$ and $D_{34}^{34}$ are invertible (these are the quantum determinants (5.9)). One can compute explicitly the inverse 
change of variables,

$$
\begin{aligned}
& x=\left(\begin{array}{ll}
g_{11} & g_{12} \\
g_{21} & g_{22}
\end{array}\right), \quad t=\left(\begin{array}{cc}
-q^{-1} D_{23} D_{12}^{-1} & D_{13} D_{12}^{-1}, \\
-q^{-1} D_{24} D_{12}^{-1} & D_{14} D_{12}^{-1}
\end{array}\right) \\
& y=\left(\begin{array}{ll}
g_{33} & g_{34} \\
g_{43} & g_{44}
\end{array}\right), \quad d=g_{55}, \\
& \tilde{\tau}=\left(\begin{array}{ll}
g_{55}^{-1} \gamma_{51} & g_{55}^{-1} \gamma_{51}
\end{array}\right), \quad \xi=\left(\begin{array}{ll}
g_{55}^{-1} \gamma_{53} & g_{55}^{-1} \gamma_{54}
\end{array}\right), \\
& \rho=y^{-1}\left(\begin{array}{c}
\gamma_{35} \\
\gamma_{45}
\end{array}\right)=D_{34}^{341}\left(\begin{array}{cc}
g_{44} & -q^{-1} g_{34} \\
-q g_{43} & g_{33}
\end{array}\right)=\left(\begin{array}{c}
D_{34}^{34-1} \gamma_{35} \\
D_{34}^{34} \gamma_{45}^{-1}
\end{array}\right) .
\end{aligned}
$$

It is not hard to see that $\mathcal{O}\left(P_{l, q}\right)$ is also generated by $x, y, d, \eta, \xi$ and $\tilde{\tau}$.

The quantum Poincaré supergroup times dilations is the quotient of $\mathcal{O}\left(P_{l, q}\right)$ by the ideal $\xi=0$. One can also check that it is a Hopf ideal, so the comultiplication goes to the quotient. The quantum Poincaré supergroup times dilations is then generated by the images in the quotient of $x, y, d, \eta$ and $\tilde{\tau}$. Here, Remark 6.1 applies as well. In matrix form, one has

$$
\left(\begin{array}{ccc}
x & 0 & 0 \\
t x & y & y \eta \\
\tilde{\tau} x & 0 & d
\end{array}\right) .
$$

Definition 6.1. We define the quantum big cell $\mathcal{O}_{q}\left(U_{12}\right)$ as the subring of $\mathcal{O}\left(P_{l q}\right)$ generated by $t$ and $\tilde{\tau}$.

We compute now the quantum commutation relations among the generators of the quantum big cell.

Proposition 6.4. The quantum big cell superring $\mathcal{O}_{q}\left(U_{12}\right)$ has the following presentation:

$$
\mathcal{O}_{q}\left(U_{12}\right):=\mathbb{C}_{q}\left\langle t_{i j}, \tilde{\tau}_{k l}\right\rangle / I_{U}
$$

where $I_{U}$ is the ideal generated by the relations:

$$
\begin{gathered}
t_{i 1} t_{i 2}=q t_{i 2} t_{i 1}, \quad t_{3 j} t_{4 j}=q^{-1} t_{4 j} t_{3 j}, \quad 1 \leq j \leq 2, \quad 3 \leq i \leq 4, \\
t_{31} t_{42}=t_{42} t_{31}, \quad t_{32} t_{41}=t_{41} t_{32}+\left(q^{-1}-q\right) t_{42} t_{31}, \\
\tilde{\tau}_{51} \tilde{\tau}_{52}=-q^{-1} \tilde{\tau}_{52} \tilde{\tau}_{51}, \quad t_{i j} \tilde{\tau}_{5 j}=q^{-1} \tilde{\tau}_{5 j} t_{i j}, \quad 1 \leq j \leq 2, \\
t_{i 1} \tilde{\tau}_{52}=\tilde{\tau}_{52} t_{i 1}, \quad t_{i 2} \tilde{\tau}_{51}=\tilde{\tau}_{51} t_{i 2}+\left(q^{-1}-q\right) t_{i 1} \tilde{\tau}_{52} .
\end{gathered}
$$

Proof. Direct check. 
As in the classical setting we have the following proposition.

Proposition 6.5. The quantum big cell $\mathcal{O}_{q}\left(U_{12}\right)$ admits a coaction of $\mathcal{O}\left(P_{l, q}\right)$ obtained by restricting suitably the comultiplication in $\mathcal{O}\left(P_{l, q}\right)$. Explicitly (see (6.5)),

$$
\begin{aligned}
\tilde{\Delta} t_{i j} & =t_{i j} \otimes 1+y_{i a} S(x)_{b j} \otimes t_{a b}+y_{i} \eta_{a} S(x)_{b j} \otimes \tilde{\tau}_{j b}, \\
\tilde{\Delta} \tilde{\tau}_{j} & =(d \otimes 1)\left(\tau_{a} \otimes 1+\xi_{b} \otimes t_{b a}+1 \otimes \tilde{\tau}_{a}\right)\left(S(x)_{a j} \otimes 1\right) .
\end{aligned}
$$

by choosing as before the set of generators $x, y, t, d, \tau, \rho$ and $\xi$ for $\mathcal{O}\left(P_{l, q}\right)$ and $t, \tilde{\tau}$ for $\mathcal{O}_{q}\left(U_{12}\right)$ with $d \tau=\tilde{\tau} x$.

Proof. This is so because the comultiplication is the same in the classical and the quantum group, given essentially by matrix multiplication. One has to be careful, though, when expressing the comultiplication in terms of the new generators, since the ordering appearing in the Definition (6.7) has to be kept consistently.

\section{Conclusions}

In this paper, we have obtained a quantum chiral conformal superspace as a noncommutative superalgebra that admits the action of the superconformal group $\mathrm{SL}_{q}(4 \mid 1)$. The quantum chiral Minkowski superspace is realized as the big cell inside the quantum conformal superspace, and the quantum super Poincaré group is properly defined as a sub supergroup of the conformal supergroup that preserves the bigcell.

In particular, we have used the nonobvious property of the chiral conformal superspace, the super Grassmannian of $2 \mid 0$-planes inside $\mathbb{C}^{4 \mid 1}$, of having an embedding in a superprojective space. The quantization has been performed using explicitly this embedding, thus giving implicitly a deformation of the projective superspace perhaps to be compared with the one in [23].

To obtain a supermanifold, which admits the correct real form of the Minkowski and conformal superspaces, one has to go to a larger supergeometric object, namely the flag supermanifold $F(2|0,2| 1,4 \mid 1)[1,27,28]$. Luckily enough, this superflag is also projective (which is not true for an arbitrary superflag, contrary to the nonsuper case), so the same method employed here to quantize the Grassmannian can be used for the superflag. This, however, is work that has its own peculiarities and that we leave for a further paper. 
From the physical point of view, this opens the possibility of constructing supersymmetric field theories with chiral superfields on genuinely noncommutative superspaces which carry an undeformed action of the supersymmetry algebra.

\section{Acknowledgments}

We want to thank V. S. Varadarajan for his help in the elaboration of the paper and for his hospitality at the Department of Mathematics at UCLA. We also wish to thank prof. L. Migliorini for helpful comments. D. Cervantes wants to thank the Departament de Física Teòrica, Universitat de València for the hospitality during the elaboration of this work. M. A. Lledó wants to thank the Università degli Studi di Bologna for its hospitality during the realization of this work. This work has been supported in part by grants FIS2008-06078-C03-02 and FPA2008-03811-E/INFN of Ministerio de Ciencia e Innovación (Spain) and ACOMP/2010/213 form Generalitat Valenciana.

\section{Appendix A Supergeometry}

In this appendix, we want to recall some basic definitions and facts in supergeometry. For more details see $[3,5,22,28,37]$.

\section{A.1 Basic definitions}

For definiteness, we take the ground field to be $k=\mathbb{R}, \mathbb{C}$. A superalgebra $A$ is a $\mathbb{Z}_{2}$-graded algebra, $A=A_{0} \oplus A_{1}$. $A_{0}$ is an algebra, while $A_{1}$ is an $A_{0}$-module. Let $p(x)$ denote the parity of a homogeneous element $x$,

$$
p(x)=0, \quad \text { if } x \in A_{0}, \quad p(x)=1, \quad \text { if } x \in A_{1} .
$$

The superalgebra $A$ is said to be commutative if for any two homogeneous elements $x, y$

$$
x y=(-1)^{p(x) p(y)} y x .
$$

The category of commutative superalgebras will be denoted by (salg). We call $A_{r}=A / I_{\text {odd }}$, with $I_{\text {odd }}$ the (two-sided) ideal generated by the odd nilpotents the, reduced algebra associated with $A$. Note that $A_{r}$ may have even nilpotents, making the terminology a bit awkward. 
From now on all superalgebras are assumed to be commutative unless otherwise specified.

Definition A.1. A superspace $S=\left(|S|, \mathcal{O}_{S}\right)$ is a topological space $|S|$ endowed with a sheaf of superalgebras $\mathcal{O}_{S}$ such that the stalk at a point $x \in|S|$ denoted by $\mathcal{O}_{S, x}$ is a local superalgebra for all $x \in|S|$, i.e., it has a unique (two-sided) ideal.

Definition A.2. A morphism $\phi: S \longrightarrow T$ of superspaces is given by $\phi=$ $\left(|\phi|, \phi^{\#}\right)$, where $\phi:|S| \longrightarrow|T|$ is a map of topological spaces and $\phi^{\#}$ : $\mathcal{O}_{T} \longrightarrow \phi_{*} \mathcal{O}_{S}$ is a local sheaf morphism, that is, $\phi_{x}^{\#}\left(\mathbf{m}_{|\phi|(x)}\right)=\mathbf{m}_{x}$, where $\mathbf{m}_{|\phi|(x)}$ and $\mathbf{m}_{x}$ are the maximal ideals in the stalks $\mathcal{O}_{T,|\phi|(x)}$ and $\mathcal{O}_{S, x}$ respectively.

The most important examples of superspaces are given by supermanifolds and superschemes. Let us introduce them.

Example A.1. The superspace $\mathbb{R}^{p \mid q}$ is the topological space $\mathbb{R}^{p}$ endowed with the following sheaf of superalgebras. For any $U \subset \subset_{\text {open }} \mathbb{R}^{p}$

$$
\mathcal{O}_{\mathbb{R}^{p \mid q}}(U)=C^{\infty}\left(\mathbb{R}^{p}\right)(U) \otimes \mathbb{R}\left[\xi_{1}, \ldots, \xi_{q}\right],
$$

where $\mathbb{R}\left[\xi_{1}, \ldots, \xi_{q}\right]$ is the exterior algebra (or Grassmann algebra) generated by the $q$ variables $\xi_{1}, \ldots, \xi_{q}$.

Definition A.3. A supermanifold of dimension $p \mid q$ is a superspace $M=$ $\left(|M|, \mathcal{O}_{M}\right)$ which is locally isomorphic to the superspace $\mathbb{R}^{p \mid q}$, i.e., for all $x \in|M|$ there exist an open set $V_{x} \subset|M|$ and $U \subset \mathbb{R}^{p \mid q}$ such that:

$$
\left.\left.\mathcal{O}_{M}\right|_{V_{x}} \cong \mathcal{O}_{\mathbb{R} p \mid q}\right|_{U}
$$

Let now $S=\left(|S|, \mathcal{O}_{S}\right)$ be a superspace and let $\mathcal{O}_{S, 0}$ and $\mathcal{O}_{S, 1}$ denote the following sheaves:

$$
\mathcal{O}_{S, 0}(U):=\left(\mathcal{O}_{S}(U)\right)_{0}, \quad \mathcal{O}_{S, 1}(U):=\left(\mathcal{O}_{S}(U)\right)_{1}, \quad U \subset_{\text {open }}|S|
$$

Note that $\mathcal{O}_{S, 0}$ is a sheaf of algebras, while $\mathcal{O}_{S, 1}$ is a sheaf of $\mathcal{O}_{S, 0}$-modules. We have the following.

Definition A.4. A superscheme $S$ is a superspace $\left(|S|, \mathcal{O}_{S}\right)$ such that $\mathcal{O}_{S, 1}$ is a quasi coherent sheaf of $\mathcal{O}_{S, 0}$-modules.

Morphisms of supermanifolds or of superschemes are just the morphism of the corresponding superspaces. As for supermanifolds, also superschemes can be characterized by a local model. Let us briefly describe it. 
Definition A.5. $\operatorname{Spec} A$.

Let $A$ be an object of (salg). Since $A_{0}$ is an algebra, we can consider the topological space

$$
\operatorname{Spec}\left(A_{0}\right)=\left\{\text { prime ideals } \mathbf{p} \subset A_{0}\right\}
$$

with its structural sheaf $\mathcal{O}_{A_{0}}$. The stalk $A_{\mathbf{p}}$ of the structural sheaf at the prime $\mathbf{p} \in \operatorname{Spec}\left(A_{0}\right)$ is the localization of $A_{0}$ at $\mathbf{p}$. As for any superalgebra, $A$ is a module over $A_{0}$, and we have indeed a sheaf $\widetilde{A}$ of $\mathcal{O}_{A_{0}}$-modules over Spec $A_{0}$ with stalk $A_{\mathbf{p}}$, the localization of the $A_{0}$-module $A$ over each prime $\mathbf{p} \in \operatorname{Spec}\left(A_{0}\right) . \quad \operatorname{Spec} A=\operatorname{def}\left(\operatorname{Spec} A_{0}, \widetilde{A}\right)$ is a superscheme. For more details concerning the construction of the sheaf $\widetilde{M}$ for a generic $A_{0}$ module $M$, see [35] II Section 5.

It is not hard to see that $\mathrm{Spec}$ is also a functor from the category of superalgebras to the category of superschemes. The proof is very similar to the ordinary setting (see [39] Ch. II) and can be found in [6] Ch. 10.

Example A.2. We define $\mathbf{A}^{m \mid n}:=\operatorname{Spec} k\left[x_{1}, \ldots, x_{m}, \xi_{1}, \ldots, \xi_{n}\right]$. This superscheme is called the affine superspace of dimension $m \mid n$. Its underlying scheme is the affine space $\mathbf{A}^{m}$ of dimension $m$.

A superscheme $S$ which is isomorphic to $\operatorname{Spec} A$ for some algebra $A$ is said to be an affine superscheme. When the reduced algebra $A_{r}=A / I_{\text {odd }}$ is finitely generated and reduced, that is, it has no nilpotents, we say that $\operatorname{Spec} A$ is a supervariety. We have the following.

Proposition A.1. A superspace $S$ is a superscheme if and only if it is locally isomorphic to $\operatorname{Spec} A$ for some superalgebra $A$, i.e., if for every $x \in|S|$, there

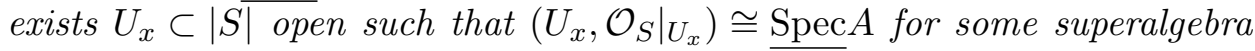
$A$ (that clearly depends on $U_{x}$ ).

Proof. See [6], Section 5.

Next, we want to introduce the concept of functor of points of a superscheme.

Definition A.6. The functor of points of a superscheme $X$ is the representable functor:

$$
\begin{array}{ccc}
h_{X}:(\text { sschemes })^{o} & \longrightarrow & (\text { sets }), \\
T & \longrightarrow h_{X}(T)=\operatorname{Hom}(T, X)
\end{array}
$$

and $h_{X}(\phi) f=f \circ \phi$ for any morphism $\phi: T \longrightarrow S$. The elements in $h_{X}(T)$ are called the T-points of $X$. 
(The label "o" means that we are taking the opposite category.)

The following facts detailed in the next observation are not difficult to prove (see Ch. 10 in ref. [6]). They will be important in the sequel.

\section{Observation A.1.}

1. The functor of points of a superscheme is determined by its restriction to the category of affine superschemes.

2. The category of affine superschemes is equivalent to the category of affine superalgebras, denoted by (salg), hence the functor of points of a superscheme can be equivalently defined also as a functor from (salg) to (sets). In other words given a superscheme $X$, we can equivalently define its functor of points as:

$$
\begin{gathered}
h_{X}:(\mathrm{salg}) \longrightarrow \\
A \longrightarrow h_{X}(A)=\operatorname{Hom}(\underline{\operatorname{Spec} A, X)}
\end{gathered}
$$

and $h_{X}(\phi) f=f \circ \underline{\text { Spec } \phi}$ for any morphism $\phi: A \longrightarrow B$.

The elements in $\overline{h_{X}}(A)$ are called the $A$-points of $X$.

When $X$ is itself an affine superscheme, the functor of points can be written as follows:

$$
\begin{array}{cc}
h_{X}:(\mathrm{salg}) \longrightarrow & (\text { sets }), \\
A & \longrightarrow h_{X}(A)=\operatorname{Hom}(\mathcal{O}(X), A)
\end{array}
$$

and $h_{X}(\phi) f=\phi \circ f$ for any morphism $\phi: A \longrightarrow B$.

Note that unless $X$ is an affine superscheme, the functor $h_{X}:(\mathrm{salg}) \longrightarrow($ sets $)$ will not be representable.

3. The functor of points of a superscheme seen as $F:($ salg $) \longrightarrow$ (sets) is a local functor, i.e., it has the sheaf property. In other words let $A \in(\mathrm{salg})$ and $\left(f_{i}, i \in I\right)=(1)=A$. Let $\phi_{k}: A \longrightarrow A_{f_{k}}$ be the natural map of the algebra $A$ to its localization and also $\phi_{k l}: A_{f_{k}} \longrightarrow A_{f_{k} f_{l}}$. Then, for a family $\alpha_{i} \in F\left(A_{f_{i}}\right)$, such that $F\left(\phi_{i j}\right)\left(\alpha_{i}\right)=F\left(\phi_{j i}\right)\left(\alpha_{j}\right)$ there exists $\alpha \in F(A)$ such that $F\left(\phi_{i}\right)(\alpha)=\alpha_{i}$.

4. (Yoneda) Given superschemes $S$ and $T$, the natural transformations $h_{S} \longrightarrow h_{T}$ are in one-to-one correspondence with the superscheme morphisms $S \longrightarrow T$. Consequently two superschemes are isomorphic if and only if their functor of points are isomorphic. 


\section{A.2 Projective supergeometry}

We want now to consider projective superschemes and supervarieties. Recall that to an ordinary graded algebra $R=\oplus_{i \geq 0} R^{i}$, we can associate the topological space

$$
\operatorname{Proj} R=\{\text { relevant homogeneous prime ideals } \mathbf{p} \subset R\}
$$

(An ideal is relevant if it does not include $R^{+}=\oplus_{i>0} R^{i}$; see [35] pg 116). The structural sheaf of $\operatorname{Proj} R$, denoted by $\mathcal{P} \mathcal{O}_{R}$ has stalk at $\mathbf{p}$ :

$$
R_{\mathbf{p}}=\left\{\frac{f}{g} \mid f \in R, g \text { homogeneous } \in R-\mathbf{p}\right\} .
$$

We want to generalize this construction to superalgebras.

Notation. We shall use the lower indices to indicate the $\mathbb{Z}_{2}$-gradation, while the upper indices will indicate the $\mathbb{Z}$-gradation. When we say "graded" we shall always mean $\mathbb{Z}$-graded, while for the $\mathbb{Z}_{2}$-graded objects we shall use the word "super".

Let us consider a graded superalgebra $A=\oplus_{i \geq 0} A^{i}$, with $\pi^{i}: A \rightarrow A^{i}$ the natural projection. We always assume that this $\mathbb{Z}$-grading is compatible with the $\mathbb{Z}_{2}$-grading $A=A_{0}+A_{1}$, i.e.,

$$
\pi^{i}\left(A_{0}\right)=A_{0} \cap A^{i} .
$$

Then we have that $A_{0}$, the even part of $A$, is a graded algebra,

$$
A_{0}=\oplus_{i \geq 0} A_{0}^{i}, \quad A_{0}^{i}=A_{0} \cap A^{i},
$$

and $A$ is a graded $A_{0}$-module.

Definition A.7. $\underline{\operatorname{Proj}} A$.

Let $A$ be a graded superalgebra. Similarly to Definition A.5 we consider the sheaf of graded superalgebras $\widetilde{A}$ on the topological space $\operatorname{Proj} A_{0}$ with stalk at $\mathbf{p} \in \operatorname{Proj} A_{0}$

$$
A_{\mathbf{p}}=\left\{\frac{f}{g} \mid f, g \in A, g \text { homogeneous } \in A_{0}-\mathbf{p}\right\} .
$$

One can check that $\left(\operatorname{Proj} A_{0}, \widetilde{A}\right)$ is a superscheme, and we will denote it with $\underline{\operatorname{Proj}} A$ (see [35] Ch. II Section 5 for more details). 
Let us see two important examples: the projective superspace and the projective supervarieties.

Example A.3. Projective superspace.

Consider the graded superalgebra $S=\mathbb{C}\left[x_{0}, \ldots, x_{m}, \xi_{1}, \ldots, \xi_{n}\right]$. We want to describe Proj $S$ explicitly as a superscheme.

For each $r, 0 \leq r \leq m$, we consider the graded superalgebra

$$
S[r]=\mathbb{C}\left[x_{0}, \ldots, x_{m}, \xi_{1}, \ldots, \xi_{n}\right]\left[x_{r}^{-1}\right], \quad \operatorname{deg}\left(x_{r}^{-1}\right)=-1 .
$$

The subalgebra $S[r]^{0} \subset S[r]$ of degree 0 is

$$
S[r]^{0} \approx \mathbb{C}\left[u_{0}, \ldots, \hat{u}_{r}, \ldots, u_{m}, \eta_{1}, \ldots, \eta_{n}\right], \quad u_{s}=\frac{x_{s}}{x_{r}}, \eta_{\alpha}=\frac{\xi_{\alpha}}{x_{r}}
$$

(the label "^" means that this generator is omitted). Let $\left|U_{r}\right|$ be the set of homogeneous prime ideals of $S[r]_{0}$. Because of (A.1), a homogeneous prime ideal of $S[r]_{0}$ corresponds to a prime ideal of the subalgebra of degree $0, S[r]_{0}^{0}$ in $S[r]^{0}=S[r]_{0}^{0} \oplus S[r]_{1}^{0}$, so $\left|U_{r}\right|=\operatorname{Spec} S[r]_{0}^{0}$. We denote by $U_{r}$ the superscheme

$$
\begin{aligned}
& U_{r}=\underline{\operatorname{Spec} S[r]^{0}} \approx\left(\operatorname{Spec} S[r]_{0}^{0}, \widetilde{S[r]^{0}}\right) \\
& \mathcal{O}_{U_{r}}={ }_{\operatorname{def}} \widetilde{S[r]^{0}}=\mathcal{O}_{\left.\mathbf{P}^{m}\right|_{\left|U_{r}\right|} \otimes \mathbb{C}\left[\xi_{1}, \ldots, \xi_{n}\right]}
\end{aligned}
$$

where $\mathbf{P}^{m}$ is the classical projective space of dimension $m$ and $\mathcal{O}_{\mathbf{P}} m$ its structural sheaf. We have then found affine open subsuperschemes $U_{r}=$ $\underline{\operatorname{Spec} S}[r]_{0}^{0}$ whose topological spaces cover Proj $S_{0}$, and

$$
\left.\mathcal{O}_{U_{r}}\right|_{\left|U_{r}\right| \cap\left|U_{s}\right|}=\left.\mathcal{O}_{U_{s}}\right|_{\left|U_{r}\right| \cap\left|U_{s}\right|}
$$

We conclude that there exists a unique sheaf on $\operatorname{Proj}(S)_{0}$ that we denote as $\mathcal{O}_{\mathbf{P}^{m} \mid n}$, whose restriction to $\left|U_{i}\right|$ is $\mathcal{O}_{U_{i}}$ and $\underline{\operatorname{Proj}} S=\left(\operatorname{Proj} S_{0}, \mathcal{O}_{\mathbf{P}^{m \mid n}}\right)$.

We will call the superscheme $\underline{\operatorname{Proj}} S=\mathbf{P}^{m \mid n}$ the projective superspace of dimension $m \mid n$.

More in general if $E$ is a super vector space and $\operatorname{Sym}(E)$ the algebra of the polynomial functions on $E$, we will denote with $\mathbf{P}(E)$ the superscheme Proj $(\operatorname{Sym}(E))$.

Example A.4. Projective supervarieties.

Let $I \subset S=\mathbb{C}\left[x_{1} \ldots x_{m}, \xi_{1} \ldots \xi_{n}\right]$ be a homogeneous ideal; then $S / I$ is also a graded superalgebra and we can consider the superscheme $\operatorname{Proj}(S / I)$. 
The natural surjective morphism $p: S \rightarrow S / I$ defines a morphism of superschemes

$$
\hat{p}: \underline{\operatorname{Proj}}(S / I) \rightarrow \underline{\operatorname{Proj}} S .
$$

Let us see this more in detail. We clearly have a morphism of the underlying topological spaces, as it happens in the classical case. In order to build a sheaf morphism, we look at an affine cover of $\operatorname{Proj}(S / I)_{0}$. We define the topological space:

$$
\begin{aligned}
\left|V_{i}\right| & =\operatorname{Proj}\left(\frac{\mathbb{C}\left[x_{0}, \ldots, x_{m}, \xi_{1}, \ldots, \xi_{n}\right]}{I}\left[x_{i}^{-1}\right]\right)_{0} \\
& =\operatorname{Spec}\left(\frac{\mathbb{C}\left[x_{0}, \ldots, x_{m}, \xi_{1}, \ldots, \xi_{n}\right]}{I}\left[x_{i}^{-1}\right]\right)_{0}^{0} \\
& =\operatorname{Spec}\left(\frac{\mathbb{C}\left[u_{0}, \ldots, \hat{u}_{i}, \ldots u_{m}, \eta_{1}, \ldots, \eta_{n}\right]}{I_{\mathrm{loc}}}\right)_{0},
\end{aligned}
$$

where $I_{\text {loc }}=(I[i])_{0}^{0} \subset \mathbb{C}\left[u_{0}, \ldots, \hat{u}_{i}, \ldots, u_{m}, \eta_{1}, \ldots, \eta_{n}\right]$ in our previous notation. We define the affine superscheme

$$
V_{i}=\underline{\operatorname{Spec}}\left(\frac{\mathbb{C}\left[u_{0}, \ldots, \hat{u}_{i}, \ldots, u_{m}, \eta_{1}, \ldots, \eta_{n}\right]}{I_{\mathrm{loc}}}\right) .
$$

One can check that the supersheaves $\mathcal{O}_{V_{i}}$ are such that $\left.\mathcal{O}_{V_{i}}\right|_{\left|V_{i}\right| \cap\left|V_{j}\right|}=$ $\left.\mathcal{O}_{V_{j}}\right|_{\left|V_{i}\right| \cap\left|V_{j}\right|}$. Hence as before there exists a supersheaf $\operatorname{Proj}(S / I)$ on $\operatorname{Proj}(S / I)_{0}$ whose restriction to $\left|V_{i}\right|$ is $\mathcal{O}_{V_{i}}$. The natural morphisms from $V_{i}$ to $\mathbf{P}^{m \mid n}$, i.e., the maps corresponding to the superalgebras morphisms:

$$
S\left[x_{i}^{-1}\right] \longrightarrow(S / I)\left[x_{i}^{-1}\right]
$$

glue accordingly to give the morphism $\hat{p}: \underline{\operatorname{Proj}}(S / I) \rightarrow \underline{\operatorname{Proj}} S$.

\section{A.3 The functor of points of projective superspace}

We now want to understand the functor of points of the projective superspace $\mathbf{P}^{m \mid n}$ and of its subvarieties. The situation is essentially the same as in the classical case. We will briefly sketch it in the super setting. (For more details of the classical case see [39] p. 111).

In Example A.3, we have given explicitly the projective superscheme $\mathbf{P}^{m \mid n}$ and an open affine covering. We want to give now explicitly its functor of 
points, namely

$$
h_{\mathbf{P}^{m \mid n}}(\underline{\operatorname{Spec} A})=\operatorname{Hom}\left(\underline{\operatorname{Spec} A}, \mathbf{P}^{m \mid n}\right), \quad A \in(\operatorname{salg}) .
$$

Using the locality property (see 3 of Facts A.1), one can prove that a morphism $\psi: \operatorname{Spec} A \longrightarrow \mathbf{P}^{m \mid n}$ is determined by a family of morphisms $\psi_{i}$ : $\underline{\operatorname{Spec}} A_{f_{i}} \longrightarrow \mathbf{P}^{m} \mid n$, where $\left(f_{i}, i \in I\right)=(1)=A$, provided they induce the same map on intersections (see Proposition III-39 in [39]). If we denote by $\phi_{k l}^{\prime}: \underline{\operatorname{Spec}} A_{f_{k} f_{l}} \longrightarrow \underline{\operatorname{Spec}} A_{f_{k}}$, the maps of superschemes induced by $\phi_{k l}$ : $A_{f_{k}} \longrightarrow \underline{\operatorname{Spec}} A_{f_{k} f_{l}}$, then we have that

$$
\psi_{i} \circ \phi_{i j}^{\prime}=\psi_{j} \circ \phi_{j i}^{\prime}
$$

We want now to give a description of the morphisms $\operatorname{Spec} A \longrightarrow \mathbf{P}^{m \mid n}$ when $A$ is a local superalgebra (Proposition III-36 in [39]).

Proposition A.2. Let $A$ be a local algebra. Then the morphisms $\operatorname{Spec} A \longrightarrow$ $\mathbf{P}^{m \mid n}$ are in one to one correspondence with the set of $(m+\overline{1 \mid n)}$-tuples $\left(a_{0}, \ldots, a_{m}, \alpha_{1}, \ldots, \alpha_{n}\right) \in A^{m+1 \mid n}$ with at least one $a_{i}$ invertible, modulo multiplication by an invertible element in $A$.

Proof. The proof is the same as the classical one, we briefly sketch it. Consider the element $\left(a_{0}, \ldots, a_{m}, \alpha_{1}, \ldots, \alpha_{n}\right) \in A^{m+1 \mid n}$ with $a_{i}$ ( $i$ fixed) a unit. We want to write the corresponding morphism of superschemes $\underline{\operatorname{Spec}} A \longrightarrow \mathbf{P}^{m \mid n}$. We can write immediately the morphism of superalgebras

$$
\begin{array}{rl}
\varphi: \quad k\left[U_{i}\right]=k\left[u_{1}, \ldots, \hat{u}_{i}, \ldots, u_{m}, \eta_{1}, \ldots, \eta_{n}\right] & \longrightarrow c \\
u_{j} & A \\
\eta_{k} & \mapsto a_{j} / a_{i} \\
& \mapsto \alpha_{k} / a_{i} .
\end{array}
$$

This is well defined with respect to the equivalence relation. Moreover, it defines a sheaf morphism $\varphi^{*}: \mathcal{O}_{U_{i}} \longrightarrow \mathcal{O}_{A}$, with $U_{i}=\operatorname{Speck}\left[U_{i}\right]$. One can check that $\left.\varphi_{i}^{*}\right|_{\left|U_{i}\right| \cap\left|U_{j}\right|}=\left.\varphi_{j}^{*}\right|_{\left|U_{i}\right| \cap\left|U_{j}\right|}$ hence the $\varphi_{i}^{*}$ define a morphism $\varphi$ : $\mathcal{O}_{\mathbf{P}^{m \mid n}} \longrightarrow \mathcal{O}_{A}$.

Conversely, assume that we have a morphism $\psi: \operatorname{Spec} A \longrightarrow \mathbf{P}^{m \mid n}$. The topological map sends the maximal ideal $\mathfrak{m}$ of $A_{0}$ into some $\left|U_{i}\right|,|\psi|(\mathfrak{m}) \in$ $\left|U_{i}\right|$. We claim that all $\operatorname{Spec} A_{0}$ is mapped inside $\left|U_{i}\right|$. In fact $|\psi|^{-1}\left(\left|U_{i}\right|\right)$ is an open set containing $\mathfrak{m}$ hence necessarily the whole $\operatorname{Spec} A_{0}$. Hence 
$|\psi|\left(\operatorname{Spec} A_{0}\right)=\left|U_{i}\right|$. The morphism $\psi$ is then given by a superalgebra map:

$$
\begin{gathered}
k\left[U_{i}\right]=k\left[x_{0} / x_{i}, \ldots, x_{m} / x_{i}, \xi_{1} / x_{i}, \ldots, \xi_{n} / x_{i}\right] \\
x_{k} / x_{i}, \xi_{\mu} / x_{i}
\end{gathered}
$$

So this map determines: $\left(b_{0}, \ldots, b_{i}=1, \ldots, b_{m}, \beta_{1}, \ldots, \beta_{n}\right)$. One can check that this is well defined. In fact if $|\psi|(\mathfrak{m})$ is also in $\left|U_{j}\right|$ we have that it corresponds to a different $m+1 \mid n$-uple which is a multiple of the previous one.

The next observation characterizes the functor of points of subvarieties of the projective superspace, that is projective supervarieties as they are defined in Example A.4.

Observation A.2. Let $X \subset \mathbf{P}^{m \mid n}$ be a projective supervariety defined by the homogeneous ideal $I=\left(f_{1}, \ldots, f_{r}\right)$. As we saw in Proposition A.2, for $A$ a local superalgebra, the $A$-points of $\mathbf{P}^{m \mid n}$ are the $(m+1 \mid n)$-tuples $\left(a_{0}, \ldots, a_{m}, \alpha_{1}, \ldots, \alpha_{n}\right) \in A^{m+1 \mid n}$ with at least one $a_{i}$ invertible. We have that such an $(m+1 \mid n)$-tuple corresponds to an $A$-point of $X$ if and only if it is a zero of all the polynomials $f_{1}, \ldots, f_{r}$. We leave this to the reader as an exercise. For more details on the ordinary setting, see [39] Ch. III.

\section{A.4 The functor of points of the Grassmannian superscheme}

In this section we want to construct the functor of points of the Grassmannian superscheme (for a general and more detailed treatment of functor of points and Grassmannian see [6] Section 3).

We want to stress the importance of the functorial treatment since it is more general and it gives geometric intuition to the problem. In fact it allows to recover the description of Grassmannian superscheme as the set of projective submodules of rank $r \mid s$ inside some free $m \mid n$-module. Let us see more in detail this construction.

Consider the functor Gr : (salg) $\longrightarrow$ (sets), with $\operatorname{Gr}(A)$ the set of projective quotients of rank $r \mid s$ of $A^{m \mid n}$, that is,

$$
\operatorname{Gr}(A)=\left\{\alpha: A^{m \mid n} \rightarrow L, \text { with } L \text { a projective A-module of rank } r \mid s\right\}
$$

where $\alpha \cong \alpha^{\prime}$ if and only if they have the same kernel. 
This functor can also be equivalently defined on the objects as the set:

$$
\operatorname{Gr}(A)=\left\{\text { projective submodules } L \text { of } A^{m \mid n} \text { of rank } r \mid s\right\}
$$

To complete the definition of functor of points we need to specify $\mathrm{Gr}$ on morphisms $\psi: A \longrightarrow B$.

Given a morphism $\psi: A \rightarrow B$ on (salg), we can give to $B$ the structure of $A$-module by setting

$$
a \cdot b=\psi(a) b, \quad a \in A, b \in B
$$

Also, given an $A$-module $L$, we can construct the $B$-module $L \otimes_{A} B$. So given $\psi$ and the element of $\operatorname{Gr}(A), f: A^{m \mid n} \rightarrow L$, we have an element of $\operatorname{Gr}(B)$

$$
\operatorname{Gr}(\psi)(f): B^{m \mid n}=A^{m \mid n} \otimes_{A} B \rightarrow L \otimes_{A} B
$$

We want to briefly motivate why Gr is the functor of points of a superscheme, sending the reader to [6] and [38] for more details and the complete proof. We use the following result [6] Section 3:

Theorem A.1. A functor $F:(\mathrm{salg}) \longrightarrow$ (sets) is the functor of points of a superscheme $X$ if and only if it has the sheaf property and it admits a cover by open affine subfunctors.

We will start by showing that Gr admits a cover by open affine subfunctors. Consider the multiindex $I=\left(i_{1}, \ldots, i_{r} \mid \mu_{1}, \ldots, \mu_{s}\right)$ and the map $\phi_{I}: A^{r \mid s} \longrightarrow A^{m \mid n}$ defined by

$$
\begin{aligned}
\phi_{I}\left(x_{1}, \ldots, x_{r} \mid \xi_{1}, \ldots, \xi_{s}\right)= & m \mid n-\text { tuple with } \\
& x_{1}, \ldots, x_{r} \text { occupying the position } i_{1}, \ldots, i_{r}, \\
& \xi_{1}, \ldots, \xi_{s} \text { occupying the position } \mu_{1}, \ldots, \mu_{s} \\
& \text { and the other positions are occupied by zero. }
\end{aligned}
$$

(For example, let $m=n=2$ and $r=s=1$. Then $\phi_{1 \mid 2}(x, \xi)=(x, 0 \mid 0, \xi)$ ).

It is possible to define subfunctors $v_{I}$ of Gr that on local superalgebras look like:

$$
v_{I}(A)=\left\{\alpha: A^{m \mid n} \longrightarrow L / \alpha \circ \phi_{I} \text { is invertible }\right\} .
$$

It turns out that the $v_{I}$ 's are representable and they correspond to the affine superspace $\mathbf{A}^{r|s \times m| n}$, moreover they cover $\mathrm{Gr}$. 
As for the sheaf property of Gr, notice that classically we have a functorial equivalence between the categories of projective finitely generated $A$ modules and coherent sheaves on $\operatorname{Spec} A$ which are locally of constant rank (see [35] p. 111). One can check that this classical equivalence translates to the super category, hence our functor can be identified with:

$$
\operatorname{Gr}(A) \cong\left\{\mathcal{F} \subset \mathcal{O}_{A}^{m \mid n} / \mathcal{F} \text { is a subsheaf, of locally constant rank } r \mid s\right\}
$$

where $\mathcal{O}_{A}^{m \mid n}=k^{m \mid n} \otimes \mathcal{O}_{A}$.

By its very definition this functor is local. Hence, we have proven that Gr is the functor of points of a superscheme, i.e., $G=h_{S}$.

\section{A.5 Actions of supergroups}

In this section, we briefly summarize the definition and main properties of the stabilizer functor for the action of a supergroup on a superscheme. Since a supervariety is in particular a superscheme, all our statements hold if we replace the word "superscheme" with "supervariety".

Definition A.8. We say that a supergroup $G$ acts on a superscheme $X$, if we have a natural transformation between their functor of points:

$$
\begin{array}{ccc}
h_{G}(A) \times h_{X}(A) & \longrightarrow & h_{X}(A), \\
g, x & \mapsto & g \cdot x
\end{array}
$$

satisfying the usual axioms for an action:

- $g \cdot(h \cdot x)=g h \cdot x$, for all $g, h \in h_{G}(A), x \in h_{X}(A)$;

- $1_{G} \cdot x=x$, for all $x \in h_{X}(A)$.

Once a supergroup action is defined, we can talk about the stabilizer of a subfunctor of $h_{X}$. We are not assuming the subfunctor to be representable or in general to be a sub-superscheme of $X$. In the following we are inspired by [36] Ch. 1, Section 2 .

Definition A.9. Let $Y$ be a subfunctor of $h_{X}$. We call Stab Sta $_{Y}$ thabilizer of $Y$ the following supergroup subfunctor of $h_{G}$ :

$$
\operatorname{Stab}_{Y}(\mathcal{A}):=\left\{g \in h_{G}(\mathcal{A}) \quad \mid \quad g \cdot Y\left(\mathcal{A}^{\prime}\right)=Y\left(\mathcal{A}^{\prime}\right) \quad \forall \mathcal{A}-\text { algebras } \quad \mathcal{A}^{\prime}\right\}
$$


Note that by this definition there is no guarantee that $\mathrm{Stab}_{Y}$ is the functor of points of a superscheme and this even in the case in which $Y$ is a subscheme of $X$. In fact, as it happens already in the ordinary setting, we have examples for $Y$ an open subscheme of $X$, for which $\mathrm{Stab}_{Y}$ is not representable. Consider for example the natural action of the multiplicative group $G(\mathcal{A})=\mathcal{A}^{1 \mid 0} \backslash\{0\}$ on $X=\mathcal{A}^{1 \mid 0}$ by multiplication and let $Y(\mathcal{A})=\mathcal{A}^{1 \mid 0} \backslash\{0\}$. This is the functor of points of an open subscheme of $X$. Geometrically it is clear that the only points stabilizing the open subset $Y(\mathcal{A})$ are the units in $G(\mathcal{A})$, that is, the elements that have inverse. However if one computes the stabilizer of $Y(\mathcal{A})$ one finds that $\operatorname{Stab}_{Y}(\mathcal{A})$ consists of the elements $f+n$, with $f$ a unit and $n$ a nilpotent element of $\mathcal{A}$. One can prove that this functor is not representable.

Despite this complication, we however have some positive answer to the question whether $\operatorname{Stab}_{Y}$ is the functor of points of a superscheme (see [6] Ch. 6 for more details and the proof of this statement).

Proposition A.3. Let the notation be as above and assume $Y$ is the functor of points of a closed subscheme of $X$. Then $\mathrm{Stab}_{Y}$ is representable and it is a closed subgroup of the supergroup $G$.

\section{References}

[1] R. Fioresi, M. A. Lledo and V. S. Varadarajan, The Minkowski and conformal superspaces, J. Math. Phys. 48 (2007), 113505.

[2] R. Penrose, Twistor algebra, J. Math. Phys. 8 (1967), 345-366.

[3] V. S. Varadarajan, Supersymmetry for mathematicians: an introduction, Courant Lecture Notes, 1, AMS (2004).

[4] A. Connes, Non Commutative Geometry, Academic Press, 1994.

[5] P. Deligne and J. Morgan, Notes on supersymmetry (following J. Bernstein), in "Quantum fields and strings. A course for mathematicians", 1, AMS, 1999.

[6] C. Carmeli, L. Caston and R. Fioresi, Mathematical foundation of supersymmetry, EMS Ser. Lect. Math., European Math. Soc., Zurich 300 pages (2011).

[7] R. Fioresi, Quantizations of flag manifolds and conformal space time, Rev. Math. Phys. 9(4) (1997), 453-465.

[8] R. Fioresi, A deformation of the big cell inside the Grassmannian manifold $G(r, n)$, Rev. Math. Phys. 11 (1999), 25-40.

[9] R. Fioresi, Quantum deformation of the flag variety, Commun. Algebra $\mathbf{2 7}(11)$ (1999), 5669-5685. 
[10] J. H. Schwarz and P. van Nieuwenhuizen, Speculations concerning a fermionic substructure of space-time, Lett. Nuovo Cim. 34 (1982), 21.

[11] P. Bouwknegt, J. G. McCarthy and P. van Nieuwenhuizen, Fusing the coordinates of quantum superspace, Phys. Lett. B 394 (1997), 82.

[12] J. de Boer, P. A. Grassi and P. van Nieuwenhuizen, Non-commutative superspace from string theory. Phys. Lett. B 574 (2003), 98-104.

[13] S. Ferrara and M. A. Lledó, Some aspects of deformations of supersymmetric field theories, J. High Energy Phys. 05(008) (2000), 1-22.

[14] N. Seiberg, Noncommutative superspace, $N=1 / 2$ supersymmetry, field theory and string theory, J. High Energy Phys. 06(010) (2003), 1-17.

[15] S. Ferrara, M. A. Lledó and O. Maciá, Supersymmetry in noncommutative superspaces, J. High Energy Phys. 09(068) (2003), 1-18.

[16] I. Bars, C. Deliduman, A. Pasqua and B. Zumino, Superstar in noncommutative superspace via covariant quantization of the superparticle. Phys. Rev. D 6 (2003), 106006.

[17] N. Hatcher, A. Restuccia and J. Stephany, The quantum algebra of superspace, Phys. Rev. D 73 (2006), 046008; Quantum algebra of $N$ superspace, Phys. Rev. D 76 (2001), 046005.

[18] E. Chang-Young, H. Kim and H. Nakajima, Noncommutative superspace and super Heisenberg group, J. High Energy Phys. 0804(004) (2008), 1-15.

[19] Y. Manin, Multiparametric quantum deformation of the general linear supergroup, Comm. Math. Phys. 123 (1989), 163-175.

[20] R. Fioresi, On algebraic supergroups and quantum deformations, J. Alg. Appl. 2(4) (2003) 403-423.

[21] R. Fioresi, Supergroups, quantum supergroups and their homogeneous spaces. Euroconference on Brane New World and Noncommutative Geometry (Torino, 2000). Modern Phys. Lett. A 16 (2001), 269-274.

[22] R. Fioresi and M. A. Lledo, On algebraic supergroups coadjoint orbits and their deformations, Comm. Math. Phys. 245 (2004), 177-200.

[23] E. Ivanov, L. Mezincescu and P. K. Townsend, Fuzzy $\mathbb{C P}^{(n \mid m)}$ as a quantum superspace, Published in "Salamanca 2003, Symmetries in gravity and field theory', 385-408, hep-th/0311159.

[24] J. Wess and B. Zumino, A Lagrangian model invariant under supergauge transformations, Phys. Lett. B 49 (1974), 52-54.

[25] J. Wess and B. Zumino, Supergauge invariant extension of quantum electrodynamics. Nucl. Phys. B 78 (1974), 1-13.

[26] S. Ferrara and B. Zumino, Supergauge invariant Yang-Mills theories, Nucl. Phys. B 79 (1974), 413-421. 
[27] M. Kotrla and J. Niederle, Supertwistors and superspace, Czech. J. Phys. B 35 (1985), 602.

[28] Y. Manin, Gauge field theory and complex geometry, Springer Verlag, 1988. (Original Russian edition in 1984).

[29] R. Fioresi, Smoothness of algebraic supervarieties and supergroups, Pac. J. Math. 234 (2008), 295-310.

[30] F. D. Grosshans, G.-C. Rota, and J. A. Stein, Invariant theory and superalgebras, CBMS Regional Conf. Series in Mathematics, Vol. 69, (1987).

[31] A. Brini, Combinatorics, superalgebras, invariant theory and representation theory, Séminaire. Lotharingien Combin., Vol. 55 (2007).

[32] N. Ciccoli, R. Fioresi and F. Gavarini, Quantization of projective homogeneous spaces and duality principle, J. Noncommut. Geom. 2 (2008), 449-496.

[33] D. Cervantes, R. Fioresi and M. A. Lledo, On chiral quantum superspaces, in 'Supersymmetry in Mathematics and Physics', eds. S. Ferrara, R. Fioresi and V.S. Varadarajan, UCLA Los Angeles, USA 2010, Springer LNM 2027, 2011, 69-99.

[34] R. Fioresi and C. Hacon, Quantum coinvariant theory for the quantum special linear group and quantum Schubert varieties, J. Algeb. 242(2) (2001), 433-446.

[35] R. Hartshorne, Algebraic geometry, GTM, Springer-Verlag, 1991.

[36] J.C. Jantzen, Representations of algebraic groups. Pure and Applied Mathematics, Academic Press, Orlando, 131, 1987.

[37] F. A. Berezin, Introduction to superanalysis. in eds. by A. A. Kirillov. D. Reidel Publishing Company, Dordrecht (Holland) 1987. With an appendix by V. I. Ogievetsky. Translated from the Russian by J. Niederle and R. Kotecký. Translation edited by Dimitri Leŭtes.

[38] M. Demazure and P. Gabriel, Groupes Algébriques, Tome 1. Mason\&Cie, éditeur. North-Holland Publishing Company, The Netherlands, 1970.

[39] D. Eisenbud and J. Harris, The geometry of schemes, Springer-Verlag, New York, 2000.

[40] W. Fulton, Young tableaux, Cambridge University Press, Cambridge, 1997.

[41] H. H. Phung, On the structure of the quantum supergroups $G L_{q}(m \mid n)$, J. Algebra 211 (1999), 363-383. 